\title{
Homogenization of Periodically Varying Coefficients in Electromagnetic Materials
}

\author{
H. T. Banks, ${ }^{1}$ V. A. Bokil, ${ }^{1}$ D. Cioranescu, ${ }^{2}$ N. L. Gibson, ${ }^{1}$ G. Griso, ${ }^{2}$ \\ and B. Miara ${ }^{3}$
}

Received January 22, 2005; accepted (in revised form) March 28, 2005; Published online March 13, 2006

In this paper, we employ the periodic unfolding method for simulating the electromagnetic field in a composite material exhibiting heterogeneous microstructures which are described by spatially periodic parameters. We consider cell problems to calculate the effective parameters for a Debye dielectric medium in the case of a circular microstructure in two dimensions. We assume that the composite materials are quasi-static in nature, i.e., the wavelength of the electromagnetic field is much larger than the relevant dimensions of the microstructure.

KEY WORDS: Homogenization; Maxwell's equations; debye dielectric materials; pulsed antenna source microwaves.

\section{INTRODUCTION}

In this article, we study the behaviour of the electromagnetic field in a material presenting heterogeneous microstructures (composite materials), which are described by spatially periodic parameters. We will subject such composite materials to electromagnetic fields generated by currents of varying frequencies. When the period of the structure is small compared to the wavelength, the coefficients in Maxwell's equations oscillate rapidly. These oscillating coefficients are difficult to treat numerically in

\footnotetext{
${ }^{1}$ Center For Research in Scientific Computation, North Carolina State University, Raleigh, NC 27695-8205, USA. E-mail: \{htbanks;vabokil;ngibson\}@ncsu.edu

${ }^{2}$ Université Pierre et Marie Curie, Laboratoire Jacques-Louis Lions, 4, Place Jussieu, 75252 Paris, Cedex 05, France. E-mail: cioran@ann.jussieu.fr; georges.griso@wanadoo.fr

${ }^{3}$ ESIEE, Laboratoire de Modélisation et Simulation Numérique, 2, boulevard Blaise Pascal, 93160 Noisy-le-Grand France. E-mail: b.miara@esiee.fr
} 
simulations. Homogenization is a process in which the composite material having a microscopic structure is replaced with an equivalent material having macroscopic, homogeneous properties. In this process of homogenization the rapidly oscillating coefficients are replaced with new effective constant coefficients. The primary objective of homogenization, i.e., of the micro-macro approach, is to replace a system with periodically varying coefficients by a limiting homogeneous system that facilitates computation. The approach that we take here is based on the periodic unfolding method presented in [8-10]. We first mention other efforts on homogenization of Maxwell's equations.

In [26], a method based on spectral expansions for Maxwell's equations is presented, which utilizes eigenvectors of the curl operators combined with the microscopic description of the material. The homogenized material is represented using mean values of only a few eigenvectors. This method relies on the material being lossless, in which case Maxwell's equations can be associated with a self-adjoint partial differential operator. However, most materials usually have losses due to a small conductivity or dispersive effects, which renders the corresponding operator in Maxwell's equations non-selfadjoint. In [25], the authors use a singular value decomposition for analysing non-selfadjoint operators that arise in Maxwell's equations. They expand the electromagnetic field in the modes corresponding to the singular values, and show that only the smallest singular values make a significant contribution to the total field when the scale is small. Using this approach they find effective, or homogenized, material parameters for Maxwell's equations when the microscopic scale becomes small compared to the scale induced by the frequencies of the imposed currents. In [13], the authors compare two different homogenization methods for Maxwell's equations in two and three-dimensions. The first method is the classical way of determining the homogenized coefficients [10], which consists of solving an elliptic problem in a unit cell. The second method based on spectral expansions is described in [26]. In [17], the author presents an overview of the homogenization of anisotropic materials at fixed frequency using the concept of two-scaled convergence $[1,21]$. The homogenized electric and magnetic parameters, the relative permittivity and the relative permeability, respectively, are found by suitable averages of the solution of a local problem in the unit cell. In [14], a homogenization technique for harmonic Maxwell equations in a composite periodic medium is presented (see also [18, 22, 27] for some other homogenization methods for Maxwell's equations).

In this paper, we use the periodic unfolding method introduced in [9] in the abstract framework of stationary elliptic equations. The study in this paper considers constitutive laws that take into account bianisotropy, chiral symmetry, thermal and memory effects. The homogenization 
procedure yields a limit constitutive law different from the original one wherein the convolution operator that accounts for memory effects is replaced by a more complex Hilbert-Schmidt operator. We refer the reader to $[6,7]$ for the relevant theory. In the following sections, we present the electromagnetic problem that is of interest to us and then set up the corresponding homogenized problem to be solved. A comparison is made between the effective parameters obtained by the exact homogenization method presented here and those computed by traditional mixture formulae, such as the Maxwell Garnett formula, the Böttcher mixture rule or Bruggeman formula, some of which are based on physical arguments [23].

Our efforts here are motivated by use of electromagnetic interrogating signals (possibly in the Terahertz range) for detection of defects $[5,15]$ in the insulating foam on the fuel tanks of the NASA space shuttles. Defects in the foam are believed to contribute to the problem of separation of the foam during liftoff, resulting in significant damage to and possibly subsequent destruction of the space vehicle itself. The low density, closed cell foam is a very complex heterogeneous material [20]. It is a polyurethanetype foam composed of five primary substances: polymeric isocyanate, a flame retardant, a surfactant, a blowing agent and a catalyst. The surfactant controls the surface tension of a liquid and thus cell formation. The blowing agent creates the foam's cellular structure by creating millions of tiny bubbles or foam cells. As a first approximation, we consider here materials with periodic gas filled cells surrounded by a matrix of polyurethane type non-magnetic material. The dielectric properties (permittivity, conductivity, etc.) vary substantially between the cellular and matrix materials, leading to highly oscillating coefficients in the Maxwell system describing propagation, reflection and dispersion of the electromagnetic fields resulting from the interrogating probes.

\section{MAXWELL'S EQUATIONS IN A CONTINUOUS MEDIUM}

We employ Maxwell's equations for a linear and isotropic medium in a form that includes terms for the electric polarization given by
(i) $\frac{\partial \mathbf{D}}{\partial t}+\mathbf{J}_{c}-\nabla \times \mathbf{H}=\mathbf{J}_{s}$ in $(0, T) \times \Omega$,
(ii) $\frac{\partial \mathbf{B}}{\partial t}+\nabla \times \mathbf{E}=0$ in $(0, T) \times \Omega$,
(iii) $\nabla \cdot \mathbf{D}=\rho$ in $(0, T) \times \Omega$,
(iv) $\nabla \cdot \mathbf{B}=0$ in $(0, T) \times \Omega$,
(v) $\mathbf{E} \times \mathbf{n}=0$ on $(0, T) \times \partial \Omega$,
(vi) $\mathbf{E}(0, \mathbf{x})=\mathbf{0}, \mathbf{H}(0, \mathbf{x})=\mathbf{0}$ in $\Omega$. 
The vector valued functions $\mathbf{E}$ and $\mathbf{H}$ represent the strengths of the electric and magnetic fields, respectively, while $\mathbf{D}$ and $\mathbf{B}$ are the electric and magnetic flux densities, respectively. The conduction current density is denoted by $\mathbf{J}_{c}$, while the source current density is given by $\mathbf{J}_{s}$. The scalar $\rho$ represents the density of free electric charges unaccounted for in the electric polarization. We assume perfect conducting boundary conditions $(1, v)$, on the boundary $\partial \Omega$, with unit outward normal $\mathbf{n}$. We also assume zero initial conditions for all the unknown fields. System (1) is completed by constitutive laws that embody the behaviour of the material in response to the electromagnetic fields. These are given in $(0, T) \times \Omega$ in the form

(i) $\mathbf{D}(t, \mathbf{x})=\epsilon_{0} \epsilon_{r}(\mathbf{x}) \mathbf{E}(t, \mathbf{x})+\mathbf{P}(t, \mathbf{x})$,

(ii) $\mathbf{B}(t, \mathbf{x})=\mu_{0} \mathbf{H}(t, \mathbf{x})$,

where $\epsilon_{0}$, and $\mu_{0}$ are the permittivity and the permeability of free space, respectively, $\epsilon_{r}$ is the relative permittivity of the medium under investigation and $\mathbf{P}$ is the media's macroscopic electric polarization.

For the media that is of interest to us, we can neglect magnetic effects; we also assume that Ohms's law governs the electric conductivity, i.e.,

$$
\mathbf{J}_{c}(t, \mathbf{x})=\sigma(\mathbf{x}) \mathbf{E}(t, \mathbf{x}) \text { in }(0, T) \times \Omega,
$$

where $\sigma$ is the conductivity of the medium. We will modify system (1) and the constitutive laws (2) by performing a change of variables that renders the system in a form that is convenient for analysis and computation. From $(1$, i) we have

$$
\frac{\partial}{\partial t}\left(\mathbf{D}+\int_{0}^{t} \mathbf{J}_{c}(s, \mathbf{x}) d s\right)-\nabla \times \mathbf{H}=\mathbf{J}_{s} \text { in }(0, T) \times \Omega .
$$

Then we define the new variable

$$
\widetilde{\mathbf{D}}(t, \mathbf{x})=\mathbf{D}(t, \mathbf{x})+\int_{0}^{t} \mathbf{J}_{c}(s, \mathbf{x}) d s .
$$

Using definition (5) in (4), we can replace Eqs. (1, i) and (1, iii) by

$$
\begin{aligned}
& \text { (i) } \frac{\partial \widetilde{\mathbf{D}}}{\partial t}-\nabla \times \mathbf{H}=\mathbf{J}_{s} \text { in }(0, T) \times \Omega, \\
& \text { (iii) } \nabla \cdot \widetilde{\mathbf{D}}=0 \text { in }(0, T) \times \Omega
\end{aligned}
$$

and obtain a modified system for the fluxes $(\tilde{\mathbf{D}}, \mathbf{B})$. We note that Eq. (6, iii) follows from the continuity equation $\frac{\partial \rho}{\partial t}+\nabla \cdot \mathbf{J}_{c}=0$, the assumption 
that $\rho(0)=0$, and the assumption that $\nabla \cdot \mathbf{J}_{s}=0$ (in the sense of distributions). The modified constitutive law (2, i) after substitution of (3) and (5) becomes

$$
\widetilde{\mathbf{D}}(t, \mathbf{x})=\epsilon_{0} \epsilon_{r}(\mathbf{x}) \mathbf{E}(t, \mathbf{x})+\int_{0}^{t} \sigma(\mathbf{x}) \mathbf{E}(s, \mathbf{x}) d s+\mathbf{P}(t, \mathbf{x}) .
$$

To describe the behaviour of the media's macroscopic electric polarization $\mathbf{P}$, we employ a general integral representation model in which the polarization explicitly depends on the past history of the electric field. This convolution model is sufficiently general to include microscopic polarization mechanisms such as dipole or orientational polarization as well as ionic and electronic polarization and other frequency dependent polarization mechanisms. The resulting constitutive law can be given in terms of a polarization or displacement susceptibility kernel $v$ as

$$
\mathbf{P}(t, \mathbf{x})=\int_{0}^{t} v(t-s, \mathbf{x}) \mathbf{E}(s, \mathbf{x}) d s .
$$

Thus the modified constitutive laws are

$$
\begin{aligned}
& \text { (i) } \mathbf{D}(t, \mathbf{x})=\epsilon_{0} \epsilon_{r}(\mathbf{x}) \mathbf{E}(t, \mathbf{x})+\int_{0}^{t}\{\sigma(\mathbf{x})+v(t-s, \mathbf{x})\} \mathbf{E}(s, \mathbf{x}) d s, \\
& \text { (ii) } \mathbf{B}(t, \mathbf{x})=\mu_{0} \mathbf{H}(t, \mathbf{x}),
\end{aligned}
$$

where, in the above and henceforth we have dropped the ${ }^{\sim}$ symbol over $\mathbf{D}$, at the same time keeping in mind that $\widetilde{\mathbf{D}}$ in definition (5) is the modified electric flux density. Let us define the vector of fields

$$
\mathbf{u}=\left(\mathbf{u}_{1}^{T}, \mathbf{u}_{2}^{T}\right)^{T}=\left(\mathbf{E}^{T}, \mathbf{H}^{T}\right)^{T} \in W^{1,1}\left(0, T ; H^{1}\left(\Omega ; \mathbb{R}^{6}\right)\right)
$$

and the operator

$$
\mathbf{L u}(t, \mathbf{x})=\left(\begin{array}{l}
\mathbf{D}(t, \mathbf{x}) \\
\mathbf{B}(t, \mathbf{x})
\end{array}\right),
$$

which from (9) can be written as

$$
\begin{aligned}
\mathbf{L u}(t, \mathbf{x})= & {\left[\begin{array}{cc}
\epsilon_{0} \epsilon_{r}(\mathbf{x}) \mathbf{I}_{3} & \mathbf{0}_{3} \\
\mathbf{0}_{3} & \mu_{0} \mathbf{I}_{3}
\end{array}\right]\left(\begin{array}{l}
\mathbf{E}(t, \mathbf{x}) \\
\mathbf{H}(t, \mathbf{x})
\end{array}\right) } \\
& +\int_{0}^{t}\left\{\left[\begin{array}{cc}
\sigma(\mathbf{x}) \mathbf{I}_{3} & \mathbf{0}_{3} \\
\mathbf{0}_{3} & \mathbf{0}_{3}
\end{array}\right]+\left[\begin{array}{cc}
v(t-s, \mathbf{x}) \mathbf{I}_{3} & \mathbf{0}_{3} \\
\mathbf{0}_{3} & \mathbf{0}_{3}
\end{array}\right]\right\}\left(\begin{array}{l}
\mathbf{E}(s, \mathbf{x}) \\
\mathbf{H}(s, \mathbf{x})
\end{array}\right) d s .
\end{aligned}
$$


We label the three $6 \times 6$ coefficient matrices in (12) as

$$
\mathbf{A}(\mathbf{x})=\left[\begin{array}{cc}
\epsilon_{0} \epsilon_{r}(\mathbf{x}) \mathbf{I}_{3} & \mathbf{0}_{3} \\
\mathbf{0}_{3} & \mu_{0} \mathbf{I}_{3}
\end{array}\right], \mathbf{B}(\mathbf{x})=\left[\begin{array}{cc}
\sigma(\mathbf{x}) \mathbf{I}_{3} & \mathbf{0}_{3} \\
\mathbf{0}_{3} & \mathbf{0}_{3}
\end{array}\right], \mathbf{C}(t, \mathbf{x})=\left[\begin{array}{cc}
v(t, \mathbf{x}) \mathbf{I}_{3} & \mathbf{0}_{3} \\
\mathbf{0}_{3} & \mathbf{0}_{3}
\end{array}\right],
$$

where, in the above definitions $\mathbf{I}_{n}$ is an $n \times n$ identity matrix and $\mathbf{0}_{n}$ is an $n \times n$ matrix of zeros, $n \in \mathbb{N}$. Using these definitions we may rewrite (12) as

$$
\mathbf{L u}(t, \mathbf{x})=\mathbf{A}(\mathbf{x}) \mathbf{u}(t, \mathbf{x})+\int_{0}^{t} \mathbf{B}(\mathbf{x}) \mathbf{u}(s, \mathbf{x}) d s+\int_{0}^{t} \mathbf{C}(t-s, \mathbf{x}) \mathbf{u}(s, \mathbf{x}) d s .
$$

Next, we define the Maxwell operator $\mathbf{M}$ as

$$
\mathbf{M u}(t, \mathbf{x})=\mathbf{M}\left(\begin{array}{l}
\mathbf{E}(t, \mathbf{x}) \\
\mathbf{H}(t, \mathbf{x})
\end{array}\right)=\left(\begin{array}{c}
\nabla \times \mathbf{H}(t, \mathbf{x}) \\
-\nabla \times \mathbf{E}(t, \mathbf{x})
\end{array}\right)
$$

and the vector $\mathbf{J}_{s}$ as

$$
\mathbf{J}_{S}(t)=-J_{S}(t) \mathbf{e}_{1},
$$

where $\mathbf{e}_{1}=(1,0,0,0,0,0)^{T} \in \mathbb{R}^{6}$, is a unit basis vector. Thus Maxwell's equation can be rewritten in the form

$$
\begin{aligned}
& \text { (i) } \frac{d}{d t} \mathbf{L u}=\mathbf{M u}+\mathbf{J}_{s}(t) \text { in }(0, T) \times \Omega, \\
& \text { (ii) } \mathbf{u}(0, \mathbf{x})=\mathbf{0} \text { in } \Omega, \\
& \text { (iii) } \mathbf{u}_{1}(t, \mathbf{x}) \times \mathbf{n}(\mathbf{x})=\mathbf{0} \text { on }(0, T) \times \partial \Omega,
\end{aligned}
$$

where $\mathbf{L}$ is the operator associated with the constitutive law (14), and $\mathbf{M}$ is the Maxwell operator (15). Note that the exterior source term $\mathbf{J}_{s}$ has only one non-zero component.

We assume that the structure that occupies the domain $\Omega$ entails periodic microstructures leading to matrices $\mathbf{A}, \mathbf{B}$ and $\mathbf{C}$ with spatially oscillatory coefficients. Specifically, we will assume that $\epsilon_{r}, \sigma$ and $\nu$ are rapidly oscillating spatial functions.

\section{THE HOMOGENIZED PROBLEM}

The theory presented in this section is based on results from [7]. We denote by $Y^{\alpha}$ the reference cell of the periodic structure that occupies $\Omega$ (see Fig. 1). The construction of the homogenized problem involves solving for the corrector subterms $\overline{\mathbf{w}}_{k}^{A} \in H_{\mathrm{per}}^{1}\left(Y ; \mathbb{R}^{2}\right), \overline{\mathbf{w}}_{k} \in W^{1,1}\left(0, T ; H_{\mathrm{per}}^{1}\right.$ 

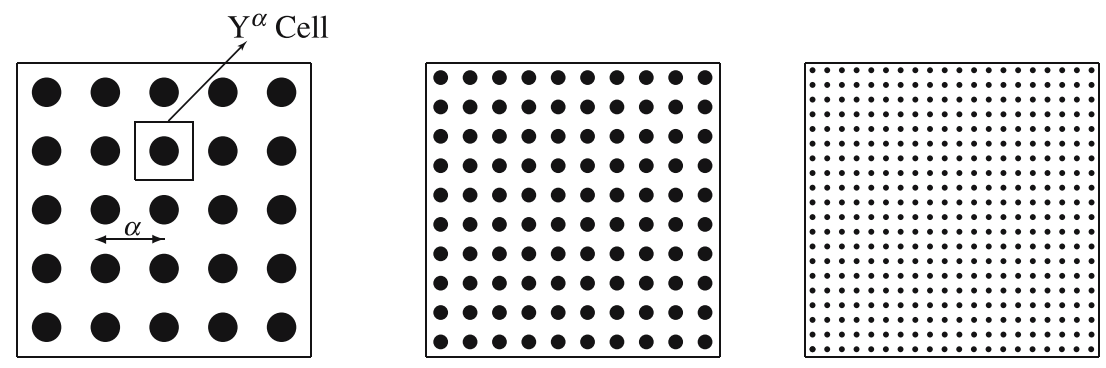

$\alpha \downarrow$

Fig. 1. Periodic composite material presenting a circular microstructure with periodicity $\alpha$. The figure shows $\alpha$ decreasing from left to right.

$\left.\left(Y ; \mathbb{R}^{2}\right)\right)$ and $\overline{\mathbf{w}}_{k}^{0} \in W^{2,1}\left(0, T ; H_{\text {per }}^{1}\left(Y ; \mathbb{R}^{2}\right)\right)$, solutions to the corrector equations

(i) $\int_{Y} \mathbf{A}(\mathbf{y}) \nabla_{\mathbf{y}} \overline{\mathbf{w}}_{k}^{A} \cdot \nabla_{\mathbf{y}} \overline{\mathbf{v}}(\mathbf{y}) d \mathbf{y}=-\int_{Y} \mathbf{A}(\mathbf{y}) \mathbf{e}_{k} \cdot \nabla_{\mathbf{y}} \overline{\mathbf{v}}(\mathbf{y}) d \mathbf{y}$,

(ii) $\int_{Y}\left\{\mathbf{A}(\mathbf{y}) \nabla_{\mathbf{y}} \overline{\mathbf{w}}_{k}(t, \mathbf{y})+\int_{0}^{t}\{\mathbf{B}(\mathbf{y})+\mathbf{C}(t-s, \mathbf{y})\} \nabla_{\mathbf{y}} \overline{\mathbf{w}}_{k}(s, \mathbf{y}) d s\right\} \cdot \nabla_{\mathbf{y}} \overline{\mathbf{v}}(\mathbf{y}) d \mathbf{y}$

$=-\int_{Y}\{\mathbf{B}(\mathbf{y})+\mathbf{C}(t, \mathbf{y})\}\left\{\mathbf{e}_{k}+\nabla_{\mathbf{y}} \overline{\mathbf{w}}_{k}^{A}\right\} \cdot \nabla_{\mathbf{y}} \overline{\mathbf{v}}(\mathbf{y}) d \mathbf{y}$

(iii) $\int_{Y}\left\{\mathbf{A}(\mathbf{y}) \nabla_{\mathbf{y}} \overline{\mathbf{w}}_{k}^{0}(t, \mathbf{y})+\int_{0}^{t}\{\mathbf{B}(\mathbf{y})+\mathbf{C}(t-s, \mathbf{y})\} \nabla_{\mathbf{y}} \overline{\mathbf{w}}_{k}^{0}(s, \mathbf{y}) d s\right\} \cdot \nabla_{\mathbf{y}} \overline{\mathbf{v}}(\mathbf{y}) d \mathbf{y}$ $=-\int_{Y} \mathbf{A}(\mathbf{y}) \mathbf{e}_{k} \cdot \nabla_{\mathbf{y}} \overline{\mathbf{v}}(\mathbf{y}) d \mathbf{y}$

for all $\overline{\mathbf{v}} \in H_{\text {per }}^{1}\left(Y ; \mathbb{R}^{2}\right)$ and $k \in\{1, \ldots, 6\}$. Here $H_{\text {per }}^{1}\left(Y ; \mathbb{R}^{2}\right)$ denotes the space of periodic functions with vanishing mean value and $\nabla_{\mathbf{y}}=\left(\nabla_{y_{1}}, \nabla_{y_{2}}\right.$, $\left.\nabla_{y_{3}}\right)^{T} \in \mathbb{R}^{3 \times 1}$. For all $\mathbf{v}=\left(v_{1}, v_{2}\right)^{T}$, with $v_{1}$ and $v_{2}$ scalar functions, we define

$$
\nabla_{\mathbf{y}} \mathbf{v}=\left(\begin{array}{c}
\nabla_{\mathbf{y}} v_{1} \\
\nabla_{\mathbf{y}} v_{2}
\end{array}\right)_{6 \times 1} \in \mathbb{R}^{6 \times 1}
$$

The first corrector term $\overline{\mathbf{u}}$, from the two-scale expansion (see [7])

$$
\mathbf{u}^{\alpha}=\mathbf{u}(\mathbf{x})+\nabla_{\mathbf{y}} \overline{\mathbf{u}}(\mathbf{x}, \mathbf{y})+\cdots, \quad \mathbf{x} \in \Omega, \mathbf{y} \in Y
$$

is given as

$$
\overline{\mathbf{u}}(t, \mathbf{x}, \mathbf{y})=\overline{\mathbf{w}}_{k}^{A}(\mathbf{y}) u_{k}(t, \mathbf{x})+\int_{0}^{t} \overline{\mathbf{w}}_{k}(t-s, \mathbf{y}) u_{k}(s, \mathbf{x}) d s+\overline{\mathbf{w}}_{k}^{0}(t, \mathbf{y}) u_{k}^{0}(\mathbf{x})
$$


with $\overline{\mathbf{u}} \in W^{2,1}\left(0, T ; H_{\text {per }}^{1}\left(Y ; \mathbb{R}^{2}\right)\right)$, where we have considered the decompositions $\mathbf{u}(t, \mathbf{x})=u_{k}(t, \mathbf{x}) \mathbf{e}_{k}$ and $\mathbf{u}^{0}(\mathbf{x})=u_{k}^{0}(\mathbf{x}) \mathbf{e}_{k}, k=1, \ldots, 6$. Rewriting (21) in matrix form we have

$$
\overline{\mathbf{u}}(t, \mathbf{x}, \mathbf{y})=\overline{\mathbf{w}}^{A}(\mathbf{y}) \mathbf{u}(t, \mathbf{x})+\int_{0}^{t} \overline{\mathbf{w}}(t-s, \mathbf{y}) \mathbf{u}(s, \mathbf{x}) d s+\overline{\mathbf{w}}^{0}(t, \mathbf{y}) \mathbf{u}^{0}(\mathbf{x}),
$$

where $\overline{\mathbf{w}}^{A} \in \mathbb{R}^{2 \times 6}$ with columns $\left\{\overline{\mathbf{w}}_{k}^{A}\right\}_{k=1}^{6}$. Similarly $\overline{\mathbf{w}}^{0}, \overline{\mathbf{w}} \in \mathbb{R}^{2 \times 6}$. The expansion (20) can be written as

$$
\begin{aligned}
& E_{x_{l}}^{\alpha}=E_{x_{l}}+\partial_{y_{l}} \bar{u}_{1}(\mathbf{x}, \mathbf{y})+\cdots, \\
& H_{x_{l}}^{\alpha}=H_{x_{l}}+\partial_{y_{l}} \bar{u}_{2}(\mathbf{x}, \mathbf{y})+\cdots,
\end{aligned}
$$

with $l=1,2,3$. We now define a new operator

$$
\mathcal{L} \mathbf{u}(t, \mathbf{x})=\mathcal{A} \mathbf{u}(t, \mathbf{x})+\mathcal{B} \int_{0}^{t} \mathbf{u}(s, \mathbf{x}) d s+\int_{0}^{t} \mathcal{C}(t-s) \mathbf{u}(s, \mathbf{x}) d s,
$$

where the $6 \times 6$ matrices $\mathcal{A}, \mathcal{B}$ and $\mathcal{C}$ are computed using the solutions of system (18) as follows

$$
\begin{aligned}
& \text { (i) } \mathcal{A}_{k}=\int_{Y} \mathbf{A}(\mathbf{y})\left\{\mathbf{e}_{k}+\nabla_{\mathbf{y}} \overline{\mathbf{w}}_{k}^{A}(\mathbf{y})\right\} d \mathbf{y}, \\
& \text { (ii) } \mathcal{B}_{k}=\int_{Y} \mathbf{B}(\mathbf{y})\left\{\mathbf{e}_{k}+\nabla_{\mathbf{y}} \overline{\mathbf{w}}_{k}^{A}(\mathbf{y})\right\} d \mathbf{y} \text {, } \\
& \text { (iii) } \mathcal{C}_{k}(t)=\int_{Y} \mathbf{C}(t, \mathbf{y})\left\{\mathbf{e}_{k}+\nabla_{\mathbf{y}} \overline{\mathbf{w}}_{k}^{A}(\mathbf{y})\right\} d \mathbf{y}+\int_{Y} \mathbf{A}(\mathbf{y}) \nabla_{\mathbf{y}} \overline{\mathbf{w}}_{k}(t, \mathbf{y}) d \mathbf{y} \\
& +\int_{Y} \int_{0}^{t}\{\mathbf{B}(\mathbf{y})+\mathbf{C}(t-s, \mathbf{y})\} \nabla_{\mathbf{y}} \overline{\mathbf{w}}_{k}(s, \mathbf{y}) d s d \mathbf{y}
\end{aligned}
$$

for $k=1,2, \ldots, 6$, and where $\mathcal{A}_{k}, \mathcal{B}_{k}, \mathcal{C}_{k}$ are the $k$ th columns of the homogenized matrices $\mathcal{A}, \mathcal{B}$ and $\mathcal{C}$, respectively. In the homogenized problem, the electromagnetic field $\mathbf{u}$ is the solution of the system

$$
\begin{aligned}
& \text { (i) } \frac{d}{d t} \mathcal{L} \mathbf{u}=\mathbf{M u}+\mathbf{J}_{s} \text { in }(0, T) \times \Omega, \\
& \text { (ii) } \mathbf{u}(0, \mathbf{x})=\mathbf{0} \text { in } \Omega, \\
& \text { (iii) } \mathbf{u}_{1}(t) \times \mathbf{n}=\mathbf{0} \text { on }(0, T) \times \partial \Omega,
\end{aligned}
$$

where $\mathbf{J}_{s}$ is as defined in (16) $\mathbf{M}$ is as defined in (15) and $\mathcal{L}$ is as defined in (24). We note that if the initial conditions are non-zero, then there is a 
supplementary source term $\mathcal{J}^{0}$ that should be introduced in the right side of $(26, \mathrm{i})$, which is given to be

$$
\mathcal{J}^{0}(t, \mathbf{x})=u_{k}^{0}(\mathbf{x}) \frac{d}{d t}\left\{\int_{Y}\left(\mathbf{A} \nabla_{\mathbf{y}} \overline{\mathbf{w}}_{k}^{0}(t)+\int_{0}^{t}(\mathbf{B}+\mathbf{C}(t-s)) \nabla_{\mathbf{y}} \overline{\mathbf{w}}_{k}^{0}(s) d s\right) d \mathbf{y}\right\},
$$

$k=1, \ldots, 6$ (see [7] for details.).

\section{REDUCTION TO TWO SPATIAL DIMENSIONS}

We now assume our problem possesses uniformity in the spatial direction $y$ (see Fig. 2 for a schematic of the computational domain). In this case Maxwell's equations decouple into two different modes, the transverse electric (TE) and transverse magnetic (TM) modes. Here, we are interested in the TE mode. The TE mode involves the components $E_{x}, E_{z}$ for the electric field and the component $H_{y}$ of the magnetic field. Let $\mathbf{x}, \mathbf{y} \in \mathbb{R}^{3}$ with $\mathbf{x}=\left(x_{1}, x_{2}, x_{3}\right)$ and $\mathbf{y}=\left(y_{1}, y_{2}, y_{3}\right)$. We will use $\mathbf{x} \in \mathbb{R}^{3}$ for points on the macro scale, and $\mathbf{y} \in \mathbb{R}^{3}$ for points on the micro scale (reference cell). Since we assume uniformity in the $x_{2}$-direction, we may take all derivatives with respect to $x_{2}$ (or $y_{2}$ in the microscale) to be zero. Then Eq. $(17, \mathrm{i}$ )

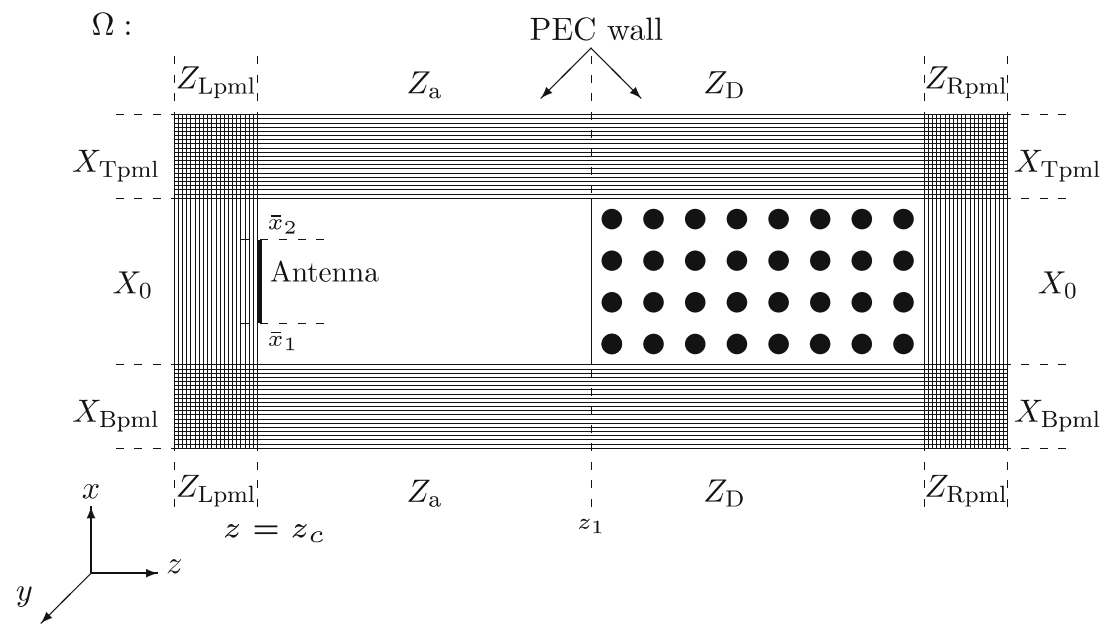

Fig. 2. The computational domain with surrounding perfectly matched absorbing layers (PML). The figure shows an antenna in the region $\left[\bar{x}_{1}, \bar{x}_{2}\right] \times z_{c}, \bar{x}_{1}, \bar{x}_{2} \in X_{0}$, and the composite material displaying periodic circular microstructures in the region $X_{0} \times Z_{D}$. The PML layers are terminated by perfectly conducting boundary conditions (PEC walls). 
when written in scalar form decouples into the TE mode

$$
\left[\begin{array}{c}
\partial_{t} D_{x_{1}} \\
\partial_{t} B_{x_{2}} \\
\partial_{t} D_{x_{3}}
\end{array}\right]=\left[\begin{array}{c}
\partial_{x_{3}} H_{x_{2}}-J_{s} \\
-\partial_{x_{3}} E_{x_{1}}+\partial_{x_{1}} E_{x_{3}} \\
\partial_{x_{1}} H_{x_{2}}
\end{array}\right]
$$

and the TM mode

$$
\left[\begin{array}{c}
\partial_{t} B_{x_{1}} \\
\partial_{t} D_{x_{2}} \\
\partial_{t} B_{x_{3}}
\end{array}\right]=\left[\begin{array}{c}
-\partial_{x_{3}} E_{x_{2}} \\
\partial_{x_{3}} H_{x_{1}}-\partial_{x_{1}} H_{x_{3}} \\
-\partial_{x_{1}} E_{x_{2}}
\end{array}\right] .
$$

Recall here that $\mathbf{D}$ is the modified electric flux density, where we have dropped the $\sim$ notation. We assume that our pulse is polarized to only have an $x_{1}$-component. In this case the component that is of interest in our problem is the $E_{x_{1}}$ component.

\section{HOMOGENIZATION MODEL IN TWO-DIMENSIONS}

In a similar manner to the three-dimensional case, we may construct matrices $\mathbf{A}^{\mathrm{TE}}, \mathbf{B}^{\mathrm{TE}}$ and $\mathbf{C}^{\mathrm{TE}}$ that represent the constitutive relations in two dimensions. Thus the constitutive matrices are

$$
\begin{aligned}
& \mathbf{A}^{\mathrm{TE}}=\left[\begin{array}{cc}
\mathbf{A}_{11}^{\mathrm{TE}} & 0 \\
0 & \mu_{0}
\end{array}\right], \quad \mathbf{B}^{\mathrm{TE}}=\left[\begin{array}{cc}
\mathbf{B}_{11}^{\mathrm{TE}} & 0 \\
0 & 0
\end{array}\right], \quad \mathbf{C}^{\mathrm{TE}}=\left[\begin{array}{cc}
\mathbf{C}_{11}^{\mathrm{TE}} & 0 \\
0 & 0
\end{array}\right], \\
& \mathbf{A}_{11}^{\mathrm{TE}}(\mathbf{x})=\left[\begin{array}{cc}
\epsilon_{0} \epsilon_{r}(\mathbf{x}) & 0 \\
0 & \epsilon_{0} \epsilon_{r}(\mathbf{x})
\end{array}\right], \quad \mathbf{B}_{11}^{\mathrm{TE}}(\mathbf{x})=\left[\begin{array}{cc}
\sigma(\mathbf{x}) & 0 \\
0 & \sigma(\mathbf{x})
\end{array}\right], \\
& \mathbf{C}_{11}^{\mathrm{TE}}(t, \mathbf{x})=\left[\begin{array}{cc}
v(t, \mathbf{x}) & 0 \\
0 & v(t, \mathbf{x})
\end{array}\right] .
\end{aligned}
$$

The homogenized solution for the TE mode is obtained from the formal asymptotic expansion (23) as

$$
\begin{aligned}
& E_{x_{1}}^{\alpha}=E_{x_{1}}+\partial_{y_{1}} \bar{u}_{1}(\mathbf{x}, \mathbf{y})+\cdots, \\
& E_{x_{3}}^{\alpha}=E_{x_{3}}+\partial_{y_{3}} \bar{u}_{1}(\mathbf{x}, \mathbf{y})+\cdots, \\
& H_{x_{2}}^{\alpha}=H_{x_{2}}+\partial_{y_{2}} \bar{u}_{2}(\mathbf{x}, \mathbf{y})+\cdots
\end{aligned}
$$


Also, since we are assuming uniformity in the $x_{2}$-direction, we set the term $\partial_{y_{2}} \bar{u}_{2}(\mathbf{x}, \mathbf{y})$ to zero. So (32) becomes

$$
\begin{aligned}
& E_{x_{1}}^{\alpha}=E_{x_{1}}+\partial_{y_{1}} \bar{u}_{1}(\mathbf{x}, \mathbf{y})+\cdots, \\
& E_{x_{3}}^{\alpha}=E_{x_{3}}+\partial_{y_{3}} \bar{u}_{1}(\mathbf{x}, \mathbf{y})+\cdots, \\
& H_{x_{2}}^{\alpha}=H_{x_{2}} .
\end{aligned}
$$

Hence the homogenized electric field for the TE mode is

$$
\mathbf{E}^{\alpha}=\mathbf{E}+\nabla_{\mathbf{y}} \bar{u}_{1}(\mathbf{x}, \mathbf{y})+\cdots,
$$

where the gradient operator in this case is $\nabla_{\mathbf{y}}=\left(\partial_{y_{1}}, \partial_{y_{3}}\right)^{T}$. Therefore, we only need to solve for $\bar{u}_{1}(\mathbf{x}, \mathbf{y})$, which in turn only depends on the first component of $\overline{\mathbf{w}}_{k}^{A}, \overline{\mathbf{w}}_{k}^{0}$ and $\overline{\mathbf{w}}_{k}$, for $k=1,2$; we will refer to these as $\bar{w}_{k}^{A}, \bar{w}_{k}^{0}$ and $\bar{w}_{k}$, respectively.

Let us again denote by $Y$ the reference cell of the periodic structure that occupies $\Omega \subset \mathbb{R}^{2}$. The construction of the two-dimensional homogenized problem involves solving for the corrector subterms $\bar{w}_{k}^{A} \in H_{\text {per }}^{1}(Y)$, $\bar{w}_{k} \in W^{1,1}\left(0, T ; H_{\mathrm{per}}^{1}(Y)\right)$ and $\bar{w}_{k}^{0} \in W^{2,1}\left(0, T ; H_{\mathrm{per}}^{1}(Y)\right)$, solutions to the corrector equations

(i) $\int_{Y} \mathbf{A}_{11}^{\mathrm{TE}}(\mathbf{y}) \nabla_{\mathbf{y}} \bar{w}_{k}^{A} \cdot \nabla_{\mathbf{y}} \bar{v}(\mathbf{y}) d \mathbf{y}=-\int_{Y} \mathbf{A}_{11}^{\mathrm{TE}}(\mathbf{y}) \mathbf{e}_{k} \cdot \nabla_{\mathbf{y}} \bar{v}(\mathbf{y}) d \mathbf{y}$,

(ii) $\int_{Y} \mathbf{A}_{11}^{\mathrm{TE}}(\mathbf{y}) \nabla_{\mathbf{y}} \bar{w}_{k}(t, \mathbf{y}) \cdot \nabla_{\mathbf{y}} \bar{v}(\mathbf{y}) d \mathbf{y}$

$$
\begin{aligned}
& +\int_{Y} \int_{0}^{t}\left\{\mathbf{B}_{11}^{\mathrm{TE}}(\mathbf{y})+\mathbf{C}_{11}^{\mathrm{TE}}(t-s, \mathbf{y})\right\} \nabla_{\mathbf{y}} \bar{w}_{k}(s, \mathbf{y}) d s \cdot \nabla_{\mathbf{y}} \bar{v}(\mathbf{y}) d \mathbf{y} \\
& =-\int_{Y}\left\{\mathbf{B}_{11}^{\mathrm{TE}}(\mathbf{y})+\mathbf{C}_{11}^{\mathrm{TE}}(t, \mathbf{y})\right\}\left\{\mathbf{e}_{k}+\nabla_{\mathbf{y}} \bar{w}_{k}^{A}\right\} \cdot \nabla_{\mathbf{y}} \bar{v}(\mathbf{y}) d \mathbf{y},
\end{aligned}
$$

(iii) $\int_{Y} \mathbf{A}_{11}^{\mathrm{TE}}(\mathbf{y}) \nabla_{\mathbf{y}} \bar{w}_{k}^{0}(t, \mathbf{y}) \cdot \nabla_{\mathbf{y}} \bar{v}(\mathbf{y}) d \mathbf{y}$

$$
\begin{aligned}
& +\int_{Y} \int_{0}^{t}\left\{\mathbf{B}_{11}^{\mathrm{TE}}(\mathbf{y})+\mathbf{C}_{11}^{\mathrm{TE}}(t-s, \mathbf{y})\right\} \nabla_{\mathbf{y}} \bar{w}_{k}^{0}(s, \mathbf{y}) d s \cdot \nabla_{\mathbf{y}} \bar{v}(\mathbf{y}) d \mathbf{y} \\
& =-\int_{Y} \mathbf{A}_{11}^{\mathrm{TE}}(\mathbf{y}) \mathbf{e}_{k} \cdot \nabla_{\mathbf{y}} \bar{v}(\mathbf{y}) d \mathbf{y}
\end{aligned}
$$

for all $\bar{v} \in H_{\mathrm{per}}^{1}(Y), k=1,2$ and $\mathbf{e}_{1}=(1,0)^{T}, \mathbf{e}_{2}=(0,1)^{T}$. Note, we drop the range space $\mathbb{R}^{n}$ in $H_{\text {per }}^{1}\left(Y ; \mathbb{R}^{n}\right)$, whenever $n=1$. Since the initial conditions that we have chosen are zero, we will not need to calculate the corrector $\bar{w}_{k}^{0}$. We will only need to solve for $\bar{w}_{k}^{A}$ and $\bar{w}_{k}$. Once we have solved 
for these corrector terms, we can then construct the homogenized matrices from

(i) $\left(\mathcal{A}_{11}^{\mathrm{TE}}\right)_{k}=\int_{Y} \mathbf{A}_{11}^{\mathrm{TE}}(\mathbf{y})\left\{\mathbf{e}_{k}+\nabla_{\mathbf{y}} \bar{w}_{k}^{A}(\mathbf{y})\right\} d \mathbf{y}$,

(ii) $\left(\mathcal{B}_{11}^{\mathrm{TE}}\right)_{k}=\int_{Y} \mathbf{B}_{11}^{\mathrm{TE}}(\mathbf{y})\left\{\mathbf{e}_{k}+\nabla_{\mathbf{y}} \bar{w}_{k}^{A}(\mathbf{y})\right\} d \mathbf{y}$,

(iii) $\left(\mathcal{C}_{11}^{\mathrm{TE}}\right)_{k}(t)=\int_{Y} \mathbf{C}_{11}^{\mathrm{TE}}(t, \mathbf{y})\left\{\mathbf{e}_{k}+\nabla_{\mathbf{y}} \bar{w}_{k}^{A}(\mathbf{y})\right\} d \mathbf{y}+\int_{Y} \mathbf{A}_{11}^{\mathrm{TE}}(\mathbf{y}) \nabla_{\mathbf{y}} \bar{w}_{k}(t, \mathbf{y}) d \mathbf{y}$

$$
+\int_{Y} \int_{0}^{t}\left\{\mathbf{B}_{11}^{\mathrm{TE}}(\mathbf{y})+\mathbf{C}_{11}^{\mathrm{TE}}(t-s, \mathbf{y})\right\} \nabla_{\mathbf{y}} \bar{w}_{k}(s, \mathbf{y}) d s d \mathbf{y},
$$

where $\mathbf{e}_{k}, k=1,2$ are the basis vectors in $\mathbb{R}^{2},\left(\mathcal{A}_{11}^{\mathrm{TE}}\right)_{k},\left(\mathcal{B}_{11}^{\mathrm{TE}}\right)_{k}$ and $\left(\mathcal{C}_{11}^{\mathrm{TE}}\right)_{k}$ are the $k$ th columns of the matrices $\mathcal{A}_{11}^{\mathrm{TE}}, \mathcal{B}_{11}^{\mathrm{TE}}$ and $\mathcal{C}_{11}^{\mathrm{TE}}$, respectively, and the homogenized matrices are given by

$$
\mathcal{A}^{\mathrm{TE}}=\left[\begin{array}{cc}
\mathcal{A}_{11}^{\mathrm{TE}} & 0 \\
0 & \mu_{0}
\end{array}\right], \quad \mathcal{B}^{\mathrm{TE}}=\left[\begin{array}{cc}
\mathcal{B}_{11}^{\mathrm{TE}} & 0 \\
0 & 0
\end{array}\right], \quad \mathcal{C}^{\mathrm{TE}}=\left[\begin{array}{cc}
\mathcal{C}_{11}^{\mathrm{TE}} & 0 \\
0 & 0
\end{array}\right] .
$$

The corresponding system of equations in the TE mode are

$$
\begin{aligned}
& \text { (i) } \frac{d}{d t} \mathcal{L}^{\mathrm{TE}} \mathbf{u}=\mathbf{M}^{\mathrm{TE}} \mathbf{u}+\mathbf{J}_{s}^{\mathrm{TE}} \text { in }(0, T) \times \Omega, \\
& \text { (ii) } \mathbf{u}(0, \mathbf{x})=\mathbf{0} \text { in } \Omega, \\
& \text { (iii) } \mathrm{u}_{3} n_{x_{1}}-\mathrm{u}_{1} n_{x_{3}}=0 \text { on }(0, T) \times \partial \Omega,
\end{aligned}
$$

where $\mathbf{u}=\left(E_{x_{1}}, E_{x_{3}}, H_{x_{2}}\right)^{T}, \mathbf{n}=\left(n_{x_{1}}, n_{x_{3}}\right)^{T}$ is the unit outward normal vector to $\partial \Omega$, the operator $\mathcal{L}^{\mathrm{TE}}$ is defined as

$$
\mathcal{L}^{\mathrm{TE}} \mathbf{u}(t, \mathbf{x})=\mathcal{A}^{\mathrm{TE}} \mathbf{u}(t, \mathbf{x})+\mathcal{B}^{\mathrm{TE}} \int_{0}^{t} \mathbf{u}(s, \mathbf{x}) d s+\int_{0}^{t} \mathcal{C}^{\mathrm{TE}}(t-s) \mathbf{u}(s, \mathbf{x}) d s
$$

and $\mathbf{M}^{\mathrm{TE}}$ is the two-dimensional curl operator

$$
\left(\begin{array}{ccc}
0 & 0 & -\partial_{x_{3}} \\
0 & 0 & \partial_{x_{1}} \\
\partial_{x_{3}} & -\partial_{x_{1}} & 0
\end{array}\right)
$$

and $\mathbf{J}_{s}^{\mathrm{TE}}(t)=-J_{s}(1,0,0)^{T}$. As mentioned in Sec. 3, if the initial conditions are non-zero, then there is a supplementary source term $\mathcal{J}^{0}$ that should be introduced in the right side of $(38, \mathrm{i})$, which is as given in (27) with $\mathbf{A}=\mathbf{A}^{\mathrm{TE}}, \mathbf{B}=\mathbf{B}^{\mathrm{TE}}$ and $\mathbf{C}=\mathbf{C}^{\mathrm{TE}}$ defined in (30) and (31). 


\section{MODELS FOR POLARIZATION}

The constitutive law in (8) is sufficiently general to include models based on differential equations and systems of differential equations or delay differential equations whose solutions can be expressed through fundamental solutions (in general variation-of-parameters representations) (see [3] for details). A number of known polarization laws can be easily treated.

1. The choice of the kernel function

$$
v(t, \mathbf{x})=\frac{\epsilon_{0}\left(\epsilon_{s}(t, \mathbf{x})-\epsilon_{\infty}(t, \mathbf{x})\right)}{\tau(t, \mathbf{x})} \mathrm{e}^{-t / \tau(t, \mathbf{x})}, \quad \mathbf{x} \in X_{0} \times Z_{D}
$$

in the dielectric $X_{0} \times Z_{D}$ (see Fig. 2) corresponds to the differential equation of the Debye Model for orientational or dipolar polarization given by

$$
\begin{aligned}
\tau(t, \mathbf{x}) \mathbf{P}_{t}(t, \mathbf{x})+\mathbf{P}(t, \mathbf{x}) & =\epsilon_{0}\left(\epsilon_{s}(t, \mathbf{x})-\epsilon_{\infty}(t, \mathbf{x})\right) \mathbf{E}(t, \mathbf{x}), \\
\mathbf{D}(t, \mathbf{x}) & =\epsilon_{0} \epsilon_{\infty}(t, \mathbf{x}) \mathbf{E}(t, \mathbf{x})+\mathbf{P}(t, \mathbf{x}) .
\end{aligned}
$$

Here $\epsilon_{s}$ is the static relative permittivity. The presence of instantaneous polarization is accounted for in this case by the coefficient $\epsilon_{\infty}$ in the electric flux equation. That is, $\epsilon_{r}=\epsilon_{\infty}$ in $X_{0} \times Z_{D}, \epsilon_{r}=1$ in air. The remainder of the electric polarization is seen to be a decaying exponential with relaxation parameter $\tau$, driven by the electric field, less the part included in the instantaneous polarization. This model was first proposed by Debye [12] to model the behaviour of materials that possess permanent dipole moments. The magnitude of the polarization term $\mathbf{P}$ represents the degree of alignment of these individual moments. The choice of coefficients in (42) gives a physical interpretation to $\epsilon_{s}$ and $\epsilon_{\infty}$ as the relative permittivities of the medium in the limit of the static field and very high frequencies, respectively. In the static case, we have $\mathbf{P}_{t}=0$, so that $\mathbf{P}=\epsilon_{0}\left(\epsilon_{s}-\epsilon_{\infty}\right) \mathbf{E}$ and $\mathbf{D}=\epsilon_{s} \epsilon_{0} \mathbf{E}$. For very high frequencies, $\tau \mathbf{P}_{t}$ dominates $\mathbf{P}$ so that $\mathbf{P} \approx 0$ and $\mathbf{D}=\epsilon_{\infty} \epsilon_{0} \mathbf{E}$.

2. The general model also includes the Lorentz model for electronic polarization which, in differential form, is represented with the second-order equation:

$$
\begin{gathered}
\mathbf{P}_{t t}(t, \mathbf{x})+\frac{1}{\tau(t, \mathbf{x})} \mathbf{P}_{t}(t, \mathbf{x})+\omega_{0}^{2} \mathbf{P}(t, \mathbf{x})=\epsilon_{0} \omega_{p}^{2}(t, \mathbf{x}) \mathbf{E}(t, \mathbf{x}), \\
\mathbf{D}(t, \mathbf{x})=\epsilon_{0} \epsilon_{\infty}(t, \mathbf{x}) \mathbf{E}(t, \mathbf{x})+\mathbf{P}(t, \mathbf{x}) .
\end{gathered}
$$


In (44), $\omega_{p}$ is called the plasma frequency and is defined to be

$$
\omega_{p}=\omega_{0} \sqrt{\epsilon_{s}-\epsilon_{\infty}},
$$

where $\omega_{0}$ is the resonance frequency. A simple variation of constants solution yields the correct kernel function

$$
\begin{gathered}
v(t)=\frac{\epsilon_{0} \omega_{p}^{2}}{\nu_{0}} \mathrm{e}^{-t / 2 \tau} \sin \left(\nu_{0} t\right), \\
v_{0}=\sqrt{\omega_{0}^{2}-\frac{1}{4 \tau^{2}}} .
\end{gathered}
$$

3. For more complex dielectric materials, a simple Debye or Lorentz polarization model is often not adequate to characterize the dispersive behaviour of the material. One can then turn to combinations of Debye, Lorentz or even more general $n$ th-order mechanisms as well as Cole-Cole type (fractional order derivatives) models. We refer the reader to $[3,11]$ for details.

\section{NUMERICAL DISCRETIZATION FOR THE CELL PROBLEM}

\subsection{Spatial Discretization Via Finite Elements}

We divide the reference cell $\mathrm{Y}$ into elementary squares, and consider $\mathcal{T}_{h}$ to be a uniform mesh with elements $\{K\}$ of edge length $h$. We define the finite dimensional space

$$
\mathbf{V}_{h}=\left\{v_{h}\left|v_{h} \in C^{0}(\overline{\mathrm{Y}}), v_{h}\right|_{K} \in Q_{1} \quad \text { for all } K \in \mathcal{T}_{h}\right\},
$$

which approximates $H^{1}(\mathrm{Y})$. In (49), the space $Q_{1}$ is defined as $Q_{1}=P_{11}$, where, for $k_{1}, k_{2} \in \mathbb{N} \cup\{0\}$

$$
P_{k_{1} k_{2}}=\left\{p\left(x_{1}, x_{2}\right) \mid p\left(x_{1}, x_{2}\right)=\sum_{0 \leqslant i \leqslant k_{1}} \sum_{0 \leqslant j \leqslant k_{2}} a_{i j} x_{1}^{i} x_{2}^{j}, a_{i j} \in \mathbb{R}\right\} .
$$

Thus, $P_{11}$ is the space of the bilinear functions of two variables, and $\mathbf{V}_{h}$ is the space of continuous piecewise bilinear functions. We now consider the subspace

$$
\begin{aligned}
& \mathbf{V}_{\text {per, h }}=\left\{v_{h} \mid v_{h} \in \mathbf{V}_{h}, v_{h}\left(y_{1}, 0\right)=v_{h}\left(y_{1}, 1\right)\right. \text { and } \\
& \left.v_{h}\left(0, y_{2}\right)=v_{h}\left(1, y_{2}\right) \text { for all } y_{1}, y_{2} \in[0,1]\right\}
\end{aligned}
$$

of $\mathbf{V}_{h}$, where $\mathrm{Y}=[0,1] \times[0,1]$. 
Thus the spatially discrete problem is to find $\bar{w}_{k, h}^{A} \in \mathbf{V}_{\text {per }, h}, \bar{w}_{k, h} \in$ $W^{1,1}\left(0, T ; \mathbf{V}_{\text {per }, h}\right)$ and $\bar{w}_{k, h}^{0} \in W^{2,1}\left(0, T ; \mathbf{V}_{\text {per }, h}\right)$, solutions to the corrector equations

(i) $\epsilon_{0} \int_{Y} \epsilon_{r, h}(\mathbf{y}) \nabla_{\mathbf{y}}^{h} \bar{w}_{k, h}^{A} \cdot \nabla_{\mathbf{y}}^{h} \bar{v}_{h}(\mathbf{y}) d \mathbf{y}=-\epsilon_{0} \int_{Y} \epsilon_{r, h}(\mathbf{y}) \mathbf{e}_{k} \cdot \nabla_{\mathbf{y}}^{h} \bar{v}_{h}(\mathbf{y}) d \mathbf{y}$,

(ii) $\epsilon_{0} \int_{Y} \epsilon_{r, h}(\mathbf{y}) \nabla_{\mathbf{y}}^{h} \bar{w}_{k, h}(t, \mathbf{y}) \cdot \nabla_{\mathbf{y}}^{h} \bar{v}_{h}(\mathbf{y}) d \mathbf{y}$

$$
\begin{aligned}
& +\int_{Y} \int_{0}^{t}\left\{\sigma_{h}(\mathbf{y})+v_{h}(t-s, \mathbf{y})\right\} \nabla_{\mathbf{y}}^{h} \bar{w}_{k, h}(s, \mathbf{y}) \cdot \nabla_{\mathbf{y}}^{h} \bar{v}_{h}(\mathbf{y}) d s d \mathbf{y} \\
& =-\int_{Y}\left\{\sigma_{h}(\mathbf{y})+v_{h}(t, \mathbf{y})\right\}\left\{\mathbf{e}_{k}+\nabla_{\mathbf{y}}^{h} \bar{w}_{k, h}^{A}\right\} \cdot \nabla_{\mathbf{y}}^{h} \bar{v}_{h}(\mathbf{y}) d \mathbf{y},
\end{aligned}
$$

(iii) $\epsilon_{0} \int_{Y} \epsilon_{r, h}(\mathbf{y}) \nabla_{\mathbf{y}}^{h} \bar{w}_{k, h}^{0}(t, \mathbf{y}) \cdot \nabla_{\mathbf{y}}^{h} \bar{v}_{h}(\mathbf{y}) d \mathbf{y}$

$$
\begin{aligned}
& +\int_{Y} \int_{0}^{t}\left\{\sigma_{h}(\mathbf{y})+v_{h}(t-s, \mathbf{y})\right\} \nabla_{\mathbf{y}}^{h} \bar{w}_{k, h}^{0}(s, \mathbf{y}) \cdot \nabla_{\mathbf{y}}^{h} \bar{v}_{h}(\mathbf{y}) d s d \mathbf{y} \\
& =-\epsilon_{0} \int_{Y} \epsilon_{r, h}(\mathbf{y}) \mathbf{e}_{k} \cdot \nabla_{\mathbf{y}}^{h} \bar{v}_{h}(\mathbf{y}) d \mathbf{y}
\end{aligned}
$$

for all $\bar{v}_{h} \in \mathbf{V}_{\text {per,h }}$ and $k=1,2$ and $\mathbf{e}_{1}=(1,0)^{T}, \mathbf{e}_{2}=(0,1)^{T}$ and $\nabla_{\mathbf{y}}^{h}$ is a discrete approximation to the gradient. In (52), we have used the definitions (31) of the matrices $\mathbf{A}_{11}^{\mathrm{TE}}, \mathbf{B}_{11}^{\mathrm{TE}}$ and $\mathbf{C}_{11}^{\mathrm{TE}}$.

\subsection{Time Discretization Via a Recursive Convolution Approach}

Since the susceptibility kernel $v(t, \mathbf{x})$, is exponential in nature for many materials of interest, we can use recursion to compute the discretized time convolution of the susceptibility kernel with the electric field in the corrector subproblems, e.g., (52, ii) and $(52$, iii). The details of the method highlighted here may be found in [2]. A similar approach known as the recursive convolution (RC) method has been used to compute the discrete convolution terms that appear in Maxwell's equations [16,19]. Let $t_{n}=n \Delta t$, for some timestep $\Delta t$ and let $\mathbf{V}^{n}$ denote the time component $\mathbf{V}(n \Delta t)$ for any vector field $\mathbf{V}$.

We will assume that all field components are constant over each time interval of length $\Delta t$. Assuming that all field vectors are zero for $t<0$, the Eq. (52, ii) can be discretized in time as 


$$
\begin{aligned}
& \epsilon_{0} \int_{Y} \epsilon_{r, h}(\mathbf{y}) \nabla_{\mathbf{y}}^{h} \bar{w}_{k, h}^{n}(\mathbf{y}) \cdot \nabla_{\mathbf{y}}^{h} \bar{v}_{h}(\mathbf{y}) d \mathbf{y} \\
& +\int_{Y} \sum_{m=0}^{n-1} \int_{m \Delta t}^{(m+1) \Delta t}\left\{\sigma_{h}(\mathbf{y})+v_{h}(n \Delta t-s, \mathbf{y})\right\} \nabla_{\mathbf{y}}^{h} \bar{w}_{k, h}^{m+1}(\mathbf{y}) \cdot \nabla_{\mathbf{y}}^{h} \bar{v}_{h}(\mathbf{y}) d s d \mathbf{y} \\
& =-\int_{Y}\left\{\sigma_{h}(\mathbf{y})+v_{h}(n \Delta t, \mathbf{y})\right\}\left\{\mathbf{e}_{k}+\nabla_{\mathbf{y}}^{h} \bar{w}_{k, h}^{A}\right\} \cdot \nabla_{\mathbf{y}}^{h} \bar{v}_{h}(\mathbf{y}) d \mathbf{y} .
\end{aligned}
$$

Let us define

$$
v_{m}^{n}(\mathbf{y})=\int_{m \Delta t}^{(m+1) \Delta t} v_{h}(n \Delta t-s, \mathbf{y}) d s
$$

Using definition (54) in (53) and rearranging we have

$$
\begin{aligned}
& \int_{Y}\left\{\epsilon_{0} \epsilon_{r, h}(\mathbf{y})+\Delta t \sigma_{h}(\mathbf{y})+v_{n-1}^{n}\right\} \nabla_{\mathbf{y}}^{h} \bar{w}_{k, h}^{n}(\mathbf{y}) \cdot \nabla_{\mathbf{y}}^{h} \bar{v}_{h}(\mathbf{y}) d \mathbf{y} \\
+ & \sum_{m=0}^{n-2} \int_{Y} v_{m}^{n} \nabla_{\mathbf{y}}^{h} \bar{w}_{k, h}^{m+1}(\mathbf{y}) \cdot \nabla_{\mathbf{y}}^{h} \bar{v}_{h}(\mathbf{y}) d \mathbf{y} \\
+ & \sum_{m=0}^{n-2} \Delta t \int_{Y} \sigma_{h}(\mathbf{y}) \nabla_{\mathbf{y}}^{h} \bar{w}_{k, h}^{m+1}(\mathbf{y}) \cdot \nabla_{\mathbf{y}}^{h} \bar{v}_{h}(\mathbf{y}) d \mathbf{y} \\
= & -\int_{Y}\left\{\sigma_{h}(\mathbf{y})+v_{h}(n \Delta t, \mathbf{y})\right\}\left\{\mathbf{e}_{k}+\nabla_{\mathbf{y}}^{h} \bar{w}_{k, h}^{A}\right\} \cdot \nabla_{\mathbf{y}}^{h} \bar{v}_{h}(\mathbf{y}) d \mathbf{y} .
\end{aligned}
$$

In Eq. (55) all the terms can be computed with the knowledge of just the solution at time $t_{n}=n \Delta t$, i.e., $\nabla_{\mathbf{y}}^{h} \bar{w}_{k, h}^{n}$, except the discrete convolution term involving $v_{m}^{n}$ for $m=0, \ldots, n-2$. We now show that the discrete convolution of all previous $\nabla_{\mathbf{y}}^{h} \bar{w}_{k, h}^{m}$ field values and the discrete susceptibility function can be reduced to recursive updating of a single vector on each element in the finite element mesh, which involves a matrix vector multiplication at each time step.

\subsection{Recursive Convolution for Debye Polarization}

We consider the case of Debye polarization in the remainder of this paper.

In this case, from (54) the function $v_{m}^{n}$ is defined as

$$
v_{m}^{n}(\mathbf{y})=\int_{m \Delta t}^{(m+1) \Delta t} \frac{\epsilon_{0}\left(\epsilon_{s, h}(\mathbf{y})-\epsilon_{\infty, h}(\mathbf{y})\right)}{\tau_{h}(\mathbf{y})} \mathrm{e}^{-\left(t_{n}-s\right) / \tau_{h}(\mathbf{y})} d s, \quad \mathbf{y} \in Y .
$$


From (55) we define the summation

$$
\Psi_{K}^{n}=\sum_{m=0}^{n-2} \int_{K} v_{m}^{n} \nabla_{\mathbf{y}}^{h} \bar{w}_{k, h}^{m+1}(\mathbf{y}) \cdot \nabla_{\mathbf{y}}^{h} \bar{v}_{h}(\mathbf{y}) d \mathbf{y}, \quad K \in \mathcal{T}_{h},
$$

where $v_{m}^{n}$ is defined in (56). Equation (57) constitutes the time discrete convolution of the susceptibility function $v_{h}$ and all field values of $\nabla_{\mathbf{y}} \bar{w}_{k, h}^{m}$ up to the $n$th time step.

Then for the $n+1$ st step we find

$$
\begin{aligned}
\Psi_{K}^{n+1}= & \sum_{m=0}^{n-2} \int_{K} v_{m}^{n+1} \nabla_{\mathbf{y}}^{h} \bar{w}_{k, h}^{m+1}(\mathbf{y}) \cdot \nabla_{\mathbf{y}}^{h} \bar{v}_{h}(\mathbf{y}) d \mathbf{y} \\
& +\int_{K} v_{n-1}^{n+1} \nabla_{\mathbf{y}}^{h} \bar{w}_{k, h}^{n}(\mathbf{y}) \cdot \nabla_{\mathbf{y}}^{h} \bar{v}_{h}(\mathbf{y}) d \mathbf{y}
\end{aligned}
$$

From (56) we can derive the identity

$$
v_{m}^{n+1}(\mathbf{y})=v_{m}^{n}(\mathbf{y}) \mathrm{e}^{-\Delta t / \tau_{h}(\mathbf{y})} .
$$

Using (59) in (58) we obtain

$$
\begin{aligned}
\Psi_{K}^{n+1}= & \sum_{m=0}^{n-2} \int_{K} v_{m}^{n} \mathrm{e}^{-\Delta t / \tau_{h}(\mathbf{y})} \nabla_{\mathbf{y}}^{h} \bar{w}_{k, h}^{m+1}(\mathbf{y}) \cdot \nabla_{\mathbf{y}}^{h} \bar{v}_{h}(\mathbf{y}) d \mathbf{y} \\
& +\int_{K} v_{n-1}^{n+1} \nabla_{\mathbf{y}}^{h} \bar{w}_{k, h}^{n}(\mathbf{y}) \cdot \nabla_{\mathbf{y}}^{h} \bar{v}_{h}(\mathbf{y}) d \mathbf{y} .
\end{aligned}
$$

In the spatial discretization of the reference cell $Y$, we will assume that the spatially dependent functions $\tau_{h}(\mathbf{y}), \epsilon_{s, h}(\mathbf{y}), \epsilon_{\infty, h}(\mathbf{y})$ and $\sigma_{h}(\mathbf{y})$ are constant on each element $K$ of the finite element mesh $\mathcal{T}_{h}$. With this assumption, for every element $K \in \mathcal{T}_{h}$, we have

$$
\begin{aligned}
\Psi_{K}^{n+1}= & \mathrm{e}^{-\Delta t / \tau_{h}(K)} \sum_{m=0}^{n-2} \int_{K} v_{m}^{n} \nabla_{\mathbf{y}}^{h} \bar{w}_{k, h}^{m+1}(\mathbf{y}) \cdot \nabla_{\mathbf{y}}^{h} \bar{v}_{h}(\mathbf{y}) d \mathbf{y} \\
& +\int_{K} v_{n-1}^{n+1} \nabla_{\mathbf{y}}^{h} \bar{w}_{k, h}^{n}(\mathbf{y}) \cdot \nabla_{\mathbf{y}}^{h} \bar{v}_{h}(\mathbf{y}) d \mathbf{y} .
\end{aligned}
$$

Combining (61) and (57), we obtain the recursion

$$
\Psi_{K}^{n+1}=\mathrm{e}^{-\Delta t / \tau_{h}(K)} \Psi_{K}^{n}+\int_{K} v_{n-1}^{n+1} \nabla_{\mathbf{y}}^{h} \bar{w}_{k, h}^{n}(\mathbf{y}) \cdot \nabla_{\mathbf{y}}^{h} \bar{v}_{h}(\mathbf{y}) d \mathbf{y},
$$


where $v_{n-1}^{n+1}(K)$, from the definition (56), can be calculated to be

$$
v_{n-1}^{n+1}(K)=\epsilon_{0}\left(\epsilon_{s, h}(K)-\epsilon_{\infty, h}(K)\right) \mathrm{e}^{(-2 \Delta t) / \tau_{h}(K)}\left(\mathrm{e}^{\Delta t / \tau_{h}(K)}-1\right) .
$$

The finite element function $\Psi_{h}^{n}$, which is defined to be $\Psi_{K}^{n}$ on element $K$ in the triangulation $\mathcal{T}_{h}$ can be calculated as

$$
\Psi_{h}^{n+1}=\sum_{K \in \mathcal{T}_{h}}\left(\mathrm{e}^{-\Delta t / \tau_{h}(K)} \Psi_{K}^{n}+\int_{K} v_{n-1}^{n+1} \nabla_{\mathbf{y}}^{h} \bar{w}_{k, h}^{n}(\mathbf{y}) \cdot \nabla_{\mathbf{y}}^{h} \bar{v}_{h}(\mathbf{y}) d \mathbf{y}\right) .
$$

Thus we have the recursion

$$
\Psi_{h}^{n+1}=\sum_{K \in \mathcal{T}_{h}} \mathrm{e}^{-\Delta t / \tau_{h}(K)} \Psi_{K}^{n}+\int_{Y} v_{n-1}^{n+1} \nabla_{\mathbf{y}}^{h} \bar{w}_{k, h}^{n}(\mathbf{y}) \cdot \nabla_{\mathbf{y}}^{h} \bar{v}_{h}(\mathbf{y}) d \mathbf{y} .
$$

We note that given the value $\Psi_{K}^{n}$ for every element $K \in \mathcal{T}_{h}$ at $t_{n}=n \Delta t$, we can calculate $\Psi_{h}^{n+1}$ by Eq. (65) which only requires the knowledge of the solution vector $\bar{w}_{k, h}^{n}$ at the $n$th step! The solutions $\bar{w}_{k, h}^{m}$ for $m<n$ are not needed to be stored in memory for this calculation. We can treat the discrete convolution in $(52$, iii) in the same manner as above.

The calculation of the discrete homogenized matrix $\mathcal{C}_{h}^{\mathrm{TE}}$ also requires the computation of a time integral which at time $t_{n}=n \Delta t$ requires the knowledge of the solutions $\bar{w}_{k, h}^{m}$ at previous times $m \Delta t, m=1, \ldots, n-1$. Thus, we again try to obtain a recursive formula that will aid in the computation of these time integrals. Once we have solved for the discrete corrector terms, we can then construct the discrete homogenized matrices from

$$
\begin{aligned}
& \text { (i) }\left(\mathcal{A}_{11, h}^{\mathrm{TE}}\right)_{k}=\int_{Y} \mathbf{A}_{11, h}^{\mathrm{TE}}(\mathbf{y})\left\{\mathbf{e}_{k}+\nabla_{\mathbf{y}}^{h} \bar{w}_{k, h}^{A}(\mathbf{y})\right\} d \mathbf{y}, \\
& \text { (ii) }\left(\mathcal{B}_{11, h}^{\mathrm{TE}}\right)_{k}=\int_{Y} \mathbf{B}_{11, h}^{\mathrm{TE}}(\mathbf{y})\left\{\mathbf{e}_{k}+\nabla_{\mathbf{y}}^{h} \bar{w}_{k, h}^{A}(\mathbf{y})\right\} d \mathbf{y} \text {, } \\
& \text { (iii) }\left(\mathcal{C}_{11, h}^{\mathrm{TE}}\right)_{k}(t)=\int_{Y} \mathbf{C}_{11, h}^{\mathrm{TE}}(t, \mathbf{y})\left\{\mathbf{e}_{k}+\nabla_{\mathbf{y}}^{h} \bar{w}_{k, h}^{A}(\mathbf{y})\right\} d \mathbf{y} \\
& +\int_{Y} \mathbf{A}_{11, h}^{\mathrm{TE}}(\mathbf{y}) \nabla_{\mathbf{y}}^{h} \bar{w}_{k, h}(t, \mathbf{y}) d \mathbf{y} \\
& +\int_{Y} \int_{0}^{t}\left\{\mathbf{B}_{11, h}^{\mathrm{TE}}(\mathbf{y})+\mathbf{C}_{11, h}^{\mathrm{TE}}(t-s, \mathbf{y})\right\} \nabla_{\mathbf{y}}^{h} \bar{w}_{k, h}(s, \mathbf{y}) d s d \mathbf{y},
\end{aligned}
$$

where as in (36), $\mathbf{e}_{k}, k=1,2$ are the basis vectors in $\mathbb{R}^{2},\left(\mathcal{A}_{11, h}^{\mathrm{TE}}\right)_{k},\left(\mathcal{B}_{11, h}^{\mathrm{TE}}\right)_{k}$ and $\left(\mathcal{C}_{11, h}^{\mathrm{TE}}\right)_{k}$ are the $k$ th columns of the discretized matrices $\mathcal{A}_{11, h}^{\mathrm{TE}}, \mathcal{B}_{11, h}^{\mathrm{TE}}$ 
and $\mathcal{C}_{11, h}^{\mathrm{TE}}$, respectively, and the discrete homogenized matrices are calculated as

$$
\mathcal{A}_{h}^{\mathrm{TE}}=\left[\begin{array}{cc}
\mathcal{A}_{11, h}^{\mathrm{TE}} & 0 \\
0 & \mu_{0}
\end{array}\right], \quad \mathcal{B}_{h}^{\mathrm{TE}}=\left[\begin{array}{cc}
\mathcal{B}_{11, h}^{\mathrm{TE}} & 0 \\
0 & 0
\end{array}\right], \quad \mathcal{C}_{h}^{\mathrm{TE}}=\left[\begin{array}{cc}
\mathcal{C}_{11, h}^{\mathrm{TE}} & 0 \\
0 & 0
\end{array}\right] .
$$

Using the definitions of the discrete matrices we rewrite (66) as

$$
\begin{aligned}
& \text { (i) }\left(\mathcal{A}_{11, h}^{\mathrm{TE}}\right)_{k}=\epsilon_{0} \int_{Y} \epsilon_{\infty, h}(\mathbf{y})\left\{\mathbf{e}_{k}+\nabla_{\mathbf{y}}^{h} \bar{w}_{k, h}^{A}(\mathbf{y})\right\} d \mathbf{y}, \\
& \text { (ii) }\left(\mathcal{B}_{11, h}^{\mathrm{TE}}\right)_{k}=\int_{Y} \sigma_{h}(\mathbf{y})\left\{\mathbf{e}_{k}+\nabla_{\mathbf{y}}^{h} \bar{w}_{k, h}^{A}(\mathbf{y})\right\} d \mathbf{y} \text {, } \\
& \text { (iii) }\left(\mathcal{C}_{11, h}^{\mathrm{TE}}\right)_{k}\left(t_{n}\right)=\int_{Y} v_{h}(t, \mathbf{y})\left\{\mathbf{e}_{k}+\nabla_{\mathbf{y}}^{h} \bar{w}_{k, h}^{A}(\mathbf{y})\right\} d \mathbf{y} \\
& +\epsilon_{0} \int_{Y} \epsilon_{\infty, h}(\mathbf{y}) \nabla_{\mathbf{y}}^{h} \bar{w}_{k, h}(t, \mathbf{y}) d \mathbf{y} \\
& +\int_{Y} \int_{0}^{n \Delta t}\left\{\sigma_{h}(\mathbf{y})+v_{h}(n \Delta t-s, \mathbf{y})\right\} \nabla_{\mathbf{y}}^{h} \bar{w}_{k, h}(s, \mathbf{y}) d s \mathrm{~d} \mathbf{y} .
\end{aligned}
$$

The term

$$
\int_{Y} \int_{0}^{n \Delta t} v_{h}(n \Delta t-s, \mathbf{y}) \nabla_{\mathbf{y}}^{h} \bar{w}_{k, h}(s, \mathbf{y}) d s d \mathbf{y}
$$

in Eq. (68, iii) can be computed using a recursive formula that does not require knowledge of the solutions $\bar{w}_{k, h}^{m}$ at times $m \Delta t, m=1, \ldots, n-1$. To derive a recursive formula, we again need to use properties of the susceptibility function $v_{h}$. We define the term in (69) to be

$$
T_{h}^{n}=\sum_{m=0}^{n-1} \int_{Y} v_{m}^{n} \nabla_{\mathbf{y}}^{h} \bar{w}_{k, h}^{m+1}(s, \mathbf{y}) d \mathbf{y} .
$$

where $v_{m}^{n}$ is defined in (56). We then have

$$
\begin{aligned}
T_{h}^{n+1} & =\sum_{m=0}^{n} \int_{Y} v_{m}^{n+1} \nabla_{\mathbf{y}}^{h} \bar{w}_{k, h}^{m+1}(s, \mathbf{y}) d \mathbf{y} \\
& =\sum_{m=0}^{n-1} \int_{Y} v_{m}^{n+1} \nabla_{\mathbf{y}}^{h} \bar{w}_{k, h}^{m+1}(s, \mathbf{y}) d \mathbf{y}+\int_{Y} v_{n}^{n+1} \nabla_{\mathbf{y}}^{h} \bar{w}_{k, h}^{n+1}(s, \mathbf{y}) d \mathbf{y} .
\end{aligned}
$$

Using (59) in (71) we obtain the recursion

$$
T_{h}^{n+1}=\sum_{K \in \mathcal{T}_{h}} \mathrm{e}^{-\Delta t / \tau(K)} T_{h}^{n}(K)+\int_{Y} v_{n}^{n+1} \nabla_{\mathbf{y}}^{h} \bar{w}_{k, h}^{n+1}(s, \mathbf{y}) d \mathbf{y} .
$$




\section{NUMERICAL EXAMPLES}

In this section, we present results of initial computations based on the above discretization schemes that we have carried out for circular microstructures. Results of numerical experiments involving square microstructures can be found in [2]. In all of these computations we have used a conjugate gradient method to solve the resulting linear systems that arise after discretizing our model in space and time.

\subsection{Examples with Varying Relative Permittivity}

In the following examples we choose the value of the relative permittivity to be

$$
\epsilon_{r}(\mathbf{x})=\epsilon_{\infty}(\mathbf{x})= \begin{cases}\epsilon_{i}=1.003, & \text { if } \mathbf{x} \in S, \\ \epsilon_{e}=2.7, & \text { if } \mathbf{x} \in Y / \bar{S},\end{cases}
$$

where $S$ is the microstructure that is enclosed inside the reference cell. These values are taken from experimental measurements for air and polyurethane material which are the primary components of the insulating foam described in the introduction.

We will solve a cell problem in the reference cell $Y=[0,1] \times[0,1]$, in which the relative permittivity is given in Eq. (73), where $S$ is the circular microstructure enclosed inside the reference cell, as depicted in Fig. 3. In this test case we will assume that $\epsilon_{s}=\epsilon_{\infty}, \sigma=0$ and $\tau=3.16 \times 10^{-8}$. Hence the parameters $\sigma$ and $\tau$ are constant over the entire dielectric material. Since $\epsilon_{\infty}=\epsilon_{s}$, we have that $v(t)=0$ for all time $t$. Thus in this example, the model possesses instant polarization but does not have a hysteretic term in the polarization. Our numerical simulation is performed on a $51 \times 51$ nodes mesh grid. We define the inclusion volume fraction $f$ as the ratio

$$
f=\frac{\text { area of inclusion }}{\text { area of domain } Y}=\text { area of inclusion. }
$$

For $f=0.5$ the homogenized matrix $\mathcal{A}_{11}^{\mathrm{TE}}$ is

$$
\mathcal{A}_{11}^{\mathrm{TE}}=\epsilon_{0}\left[\begin{array}{cc}
1.68009 & -2.92 \times 10^{-4} \\
-2.92 \times 10^{-4} & 1.68009
\end{array}\right]
$$

and the homogenized matrices $\mathcal{B}_{11}^{T E}$ and $\mathcal{C}_{11}^{T E}$ are the zero matrices.

In the numerical solution for the homogenized model we have approximated the circular microstructure in a staircase fashion. In Table I, we present the homogenized relative permittivities for different inclusion 


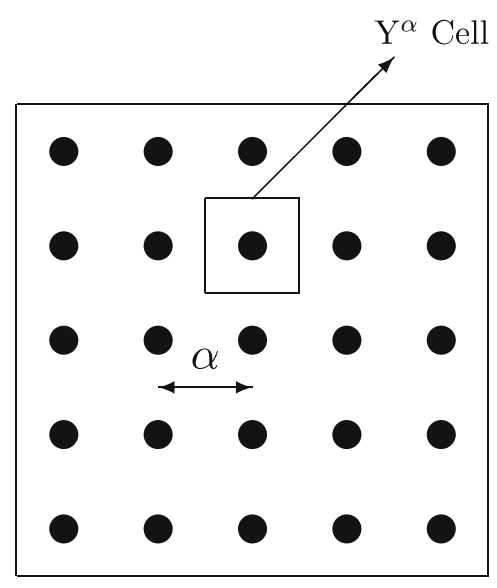

Fig. 3. (left) Periodic composite material presenting a circular microstructure with periodicity $\alpha$. (right) The reference cell $Y=[0,1] \times[0,1]$.

volume fractions $f$, and different refinements of the mesh grid that is imposed on the reference cell $Y$. Here $h$ denotes the mesh step size. In this table we note that the lower and upper off-diagonal entries, $\left(\mathcal{A}_{11}^{\mathrm{TE}}\right)_{\text {lod }}$, and $\left(\mathcal{A}_{11}^{\mathrm{TE}}\right)_{\text {uod }}$, respectively, of the homogenized matrix $\mathcal{A}_{11}^{\mathrm{TE}}$ decrease at least as fast as $\mathcal{O}(h)$ as the mesh grid is refined. The error in the off-diagonal terms is probably due to the inaccurate representation of the circular microstructure by a staircase approximation, and this is possibly also the reason for the varying magnitudes of the off-diagonal entries for different values of $f$. Hence $\mathcal{A}_{11}^{\mathrm{TE}}$ is approximately diagonal with identical diagonal entries. For the case of $f=0.5$ we have

$$
\mathcal{A}_{11}^{\mathrm{TE}} \approx 1.68 \epsilon_{0}\left[\begin{array}{cc}
1.0 & 0 \\
0 & 1.0
\end{array}\right] .
$$

Thus the homogenized or effective relative permittivity for $f=0.5$ is $\epsilon_{r}=$ 1.68 .

In Fig. 4, we plot the relative effective permittivity versus the inclusion volume fraction for the method discussed in this paper and compare it to different theoretical mixture models, namely the Maxwell-Garnett formula, the Bruggeman mixture rule, as well as the weighted average of the different relative permittivities. (We refer the reader to [23] for a discussion of the various theoretical mixing formulas.) We note how close our predicted values of the effective relative permittivity are to those predicted 
Table I. The Effective Relative Permittivities for Different Volume Fractions with a Circular Microstructure

\begin{tabular}{llllll}
\hline$f$ & $f_{\text {sc }}$ & $h$ & $\left(\mathcal{A}_{11}^{\mathrm{TE}}\right)_{\mathrm{d}}$ & \multicolumn{1}{l}{$\left(\mathcal{A}_{11}^{\mathrm{TE}}\right)_{\mathrm{lod}}$} & $\left(\mathcal{A}_{11}^{\mathrm{TE}}\right)_{\mathrm{uod}}$ \\
\hline 0.7854 & 0.78 & 0.02 & 1.23068 & $5.92955 \times 10^{-3}$ & $5.92955 \times 10^{-3}$ \\
& 0.78 & 0.01 & 1.23186 & $1.86948 \times 10^{-3}$ & $1.86948 \times 10^{-3}$ \\
& 0.785 & 0.005 & 1.23347 & $1.16484 \times 10^{-3}$ & $1.16484 \times 10^{-3}$ \\
0.5027 & 0.785 & 0.0025 & 1.23767 & $5.41779 \times 10^{-4}$ & $5.41779 \times 10^{-4}$ \\
& 0.502 & 0.02 & 1.68009 & $-2.92023 \times 10^{-4}$ & $-2.92023 \times 10^{-4}$ \\
& 0.502 & 0.01 & 1.68299 & $-7.31937 \times 10^{-5}$ & $-7.31937 \times 10^{-5}$ \\
& 0.502 & 0.005 & 1.68509 & $-1.94965 \times 10^{-5}$ & $-1.94965 \times 10^{-5}$ \\
0.7124 & 0.502 & 0.0025 & 1.68523 & $-5.02621 \times 10^{-6}$ & $-5.02621 \times 10^{-6}$ \\
& 0.7124 & 0.02 & 1.34434 & $-8.69546 \times 10^{-19}$ & $-2.42287 \times 10^{-17}$ \\
& 0.7129 & 0.01 & 1.34789 & $-3.21656 \times 10^{-18}$ & $-1.73589 \times 10^{-17}$ \\
& 0.7124 & 0.005 & 1.35081 & $2.19197 \times 10^{-16}$ & $5.59808 \times 10^{-16}$ \\
& 0.7125 & 0.0025 & 1.35216 & $-1.96101 \times 10^{-18}$ & $5.536 \times 10^{-16}$ \\
& 0.1252 & 0.02 & 2.40606 & $-6.64092 \times 10^{-4}$ & $-6.64092 \times 10^{-4}$ \\
& 0.1253 & 0.01 & 2.40637 & $-1.72749 \times 10^{-4}$ & $-1.72749 \times 10^{-4}$ \\
& 0.1255 & 0.005 & 2.40609 & $-4.39996 \times 10^{-5}$ & $-4.39996 \times 10^{-5}$ \\
& 0.1255 & 0.0025 & 2.40626 & $-1.15057 \times 10^{-5}$ & $-1.15057 \times 10^{-5}$ \\
\hline
\end{tabular}

The table lists the diagonal entries, $\left(\mathcal{A}_{11}^{\mathrm{TE}}\right)_{\mathrm{d}}$, of the homogenized matrix $\mathcal{A}_{11}^{\mathrm{TE}}$ as well as the lower and upper off-diagonal entries $\left(\mathcal{A}_{11}^{\mathrm{TE}}\right)_{\text {lod }}$ and $\left(\mathcal{A}_{11}^{\mathrm{TE}}\right)_{\text {uod }}$, respectively, for different levels of refinement of the mesh grid on the reference cell $Y$. Here $f$ is the volume fraction and $f_{\mathrm{sc}}$ is the computed volume fraction using the staircase approximation.

by these mixing rules. The prediction of the effective relative permittivity of the composite mixture $\epsilon_{\text {eff }}$ according to these formulas is given by

$$
\begin{array}{cc}
\epsilon_{\mathrm{eff}}=\epsilon_{e}+2 f \epsilon_{e} \frac{\epsilon_{i}-\epsilon_{e}}{\epsilon_{i}+\epsilon_{e}-f\left(\epsilon_{i}-\epsilon_{e}\right)} & \text { (Maxwell-Garnett), } \\
(1-f) \frac{\epsilon_{e}-\epsilon_{\mathrm{eff}}}{\epsilon_{e}+\epsilon_{\mathrm{eff}}}+f \frac{\epsilon_{i}-\epsilon_{\mathrm{eff}}}{\epsilon_{i}+\epsilon_{\mathrm{eff}}}=0 & \text { (Bruggeman). }
\end{array}
$$

These formulas hold for randomly distributed circular inclusions of permittivity $\epsilon_{i}$ in a homogeneous environment of permittivity $\epsilon_{e}$. The inclusions occupy a volume fraction $f$ of the homogeneous medium. These different mixing models predict different effective permittivity values for a given mixture. There are also bounds that limit the range of the predictions. These bounds are the Weiner bounds given by

$$
\begin{aligned}
& \epsilon_{\mathrm{eff}, \max }=f \epsilon_{i}+(1-f) \epsilon_{e} \quad \text { (weighted average) }, \\
& \epsilon_{\mathrm{eff}, \min }=\frac{\epsilon_{i} \epsilon_{e}}{f \epsilon_{e}+(1-f) \epsilon_{i}} .
\end{aligned}
$$




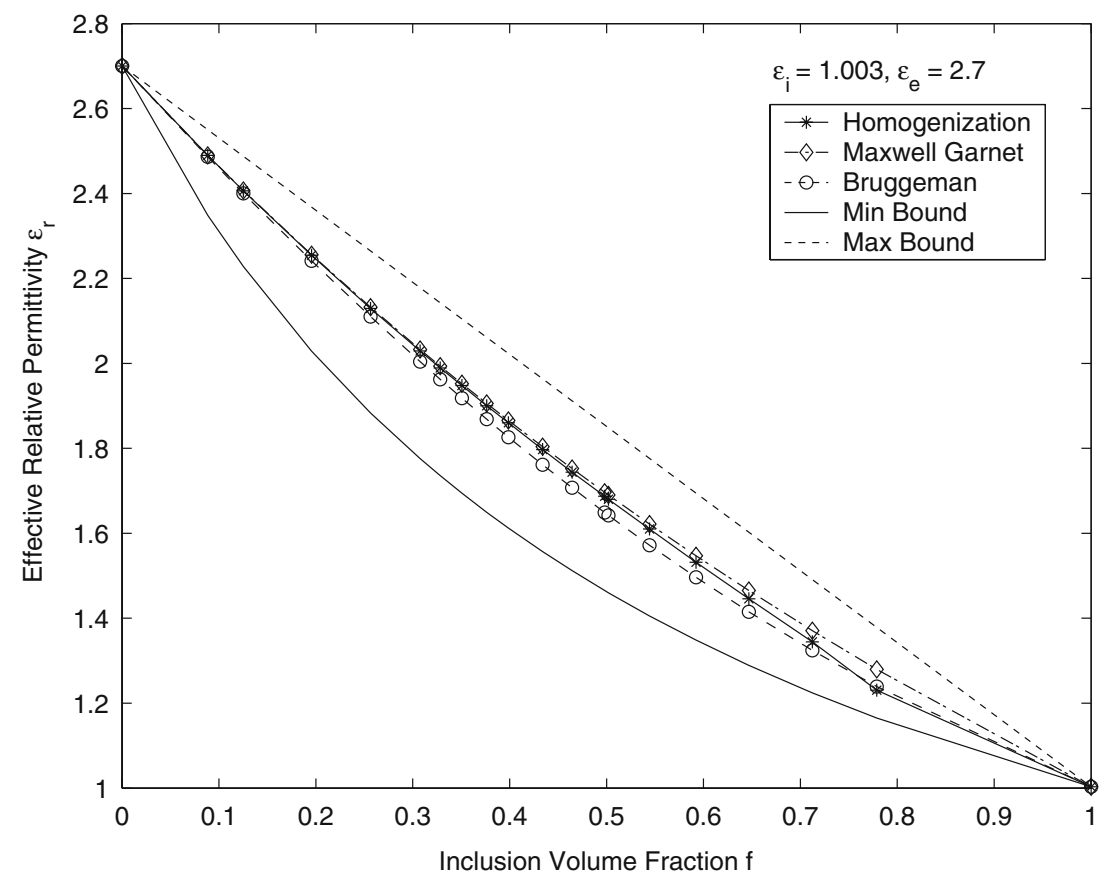

Fig. 4. Effective relative permittivity distribution for random mixtures with circular microstructures.

These bounds hold for all values of $\epsilon_{i}$ and $\epsilon_{e}$. Other mixing models such as power-law models, the Lichtenecker formula, etc., also exist in the literature. We again refer the reader to $[23,24]$ for further details. In Fig. 5, we plot the solution vectors $\bar{w}_{1}^{A}$ and $\bar{w}_{2}^{A}$, for $f=0.5$, over the reference cell $Y$. In Fig. 6 we plot a topview of the corresponding solution vectors in the domain $Y$. We observe the large deformations of the corrector functions in Figs. 5 and 6. This indicates that the contribution of these corrector functions to the electromagnetic fields is non trivial.

\subsection{Example with Varying Relaxation Times}

We now consider an example of a composite material in which the value of the relaxation time $\tau$ is given to be

$$
\tau(\mathbf{x})= \begin{cases}\tau_{i}=1.58 \times 10^{-8}, & \text { if } \mathbf{x} \in S, \\ \tau_{e}=3.16 \times 10^{-8}, & \text { if } \mathbf{x} \in Y / \bar{S},\end{cases}
$$



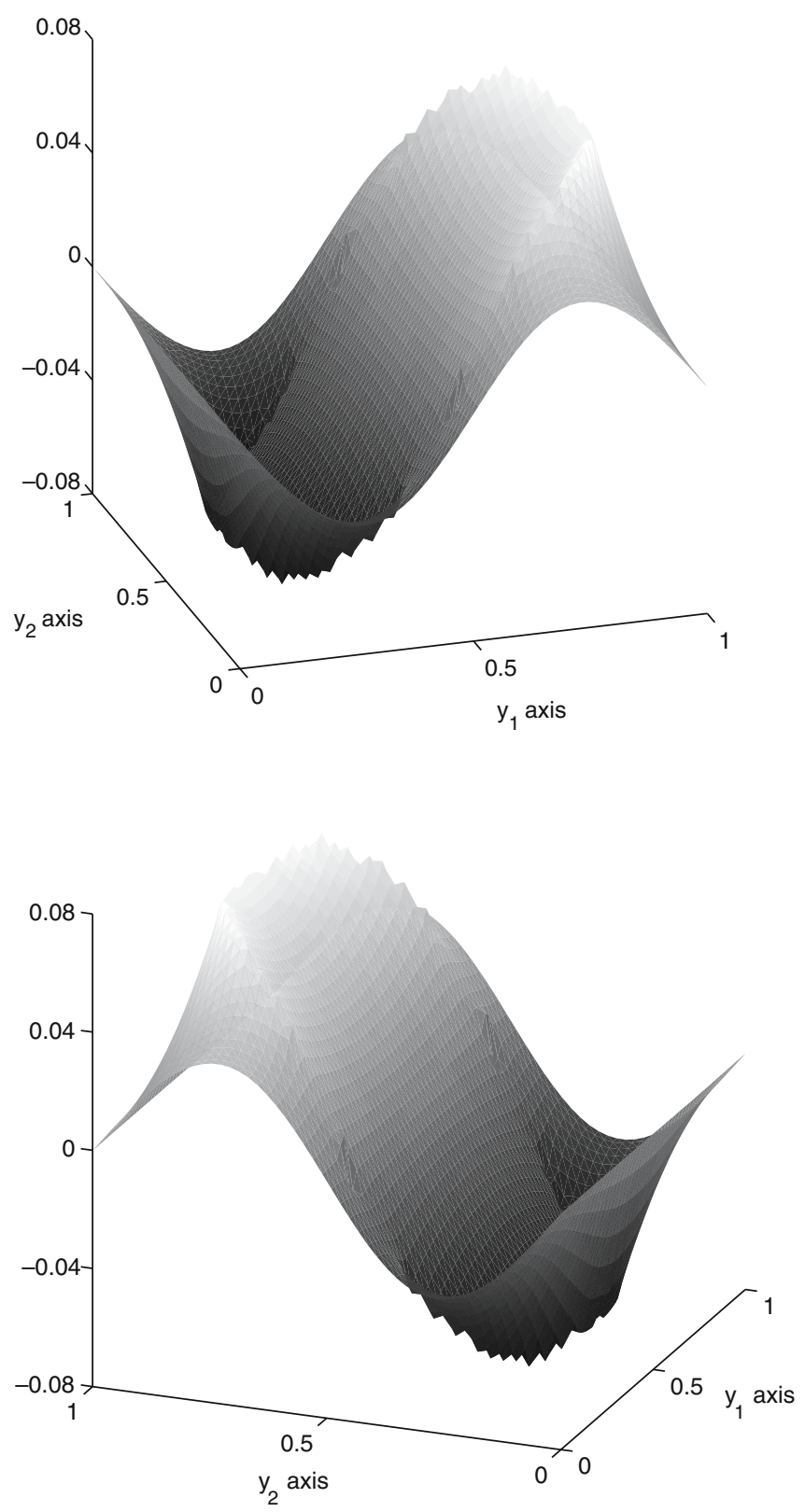

Fig. 5. Plot of the solution vectors $\bar{w}_{1}^{A}$ (top) and $\bar{w}_{2}^{A}$ (bottom) for an inclusion fraction volume $f=0.5$ on a $50 \times 50$ cells mesh grid. The homogenized value of $\epsilon_{r}$ for this case is $\approx 1.68$. 

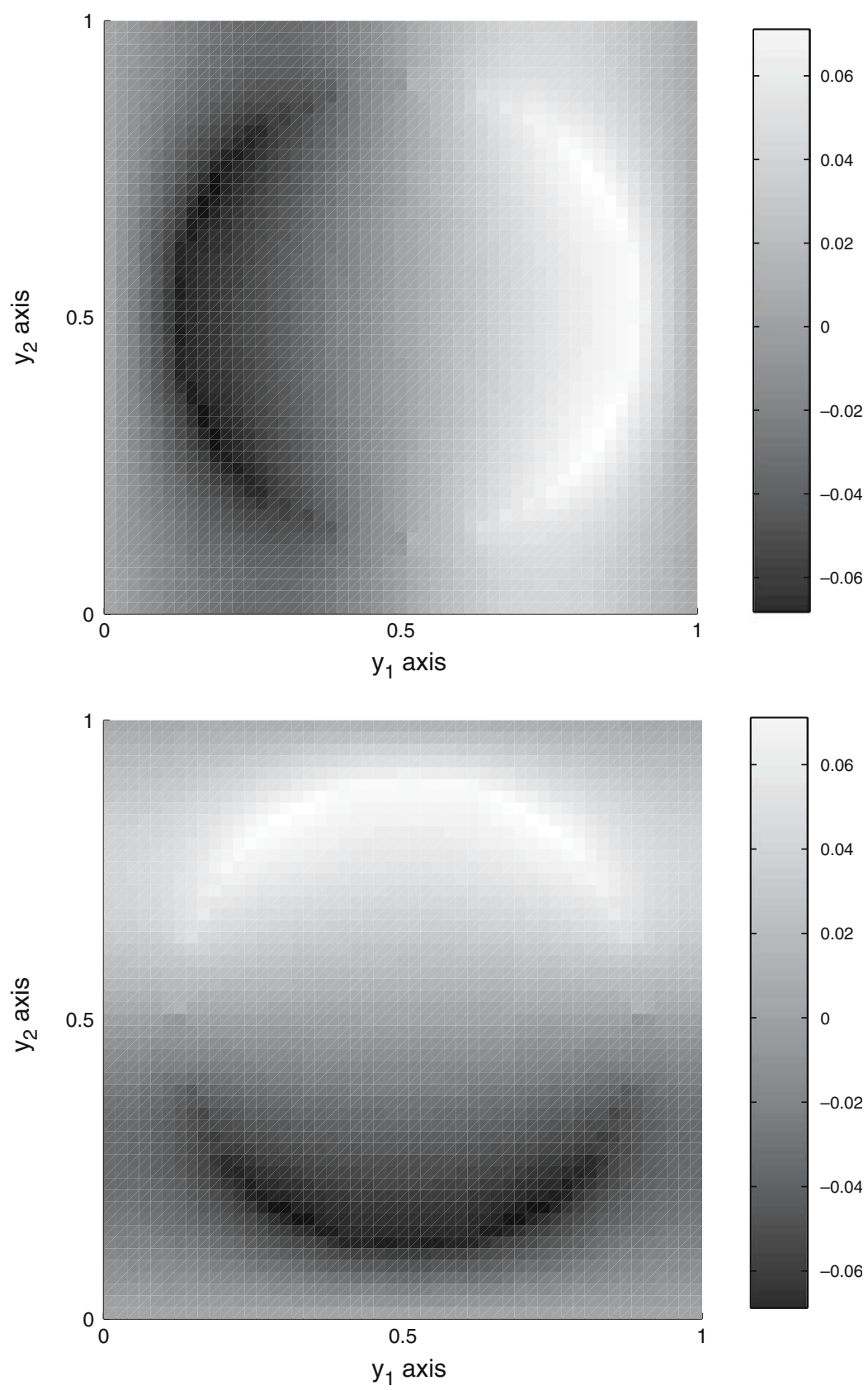

Fig. 6. Top view of the solution vectors $\bar{w}_{1}^{A}$ (top) and $\bar{w}_{2}^{A}$ (bottom). 
where again $S$ is a circular microstructure. In this test case, we will assume that the other parameters namely, $\epsilon_{s}, \sigma$ and $\epsilon_{\infty}$, are constant over the entire dielectric material with $\epsilon_{\infty}=5.5, \epsilon_{s}=78.2$ and $\sigma=1.0 \times 10^{-5}$.

In Fig. 7, we plot the results of the cell problem. This figure plots the effective dielectric response function (DRF) as a function of time. We compare the homogenized DRF with four different cases. The lowfrequency case corresponds to one in which the relaxation time $\tau$ is given to be the weighted average of $\tau_{i}$ and $\tau_{e}$

$$
\tau_{\text {low }}=f \tau_{i}+(1-f) \tau_{e} .
$$

The high-frequency case corresponds to the relaxation time

$$
\tau_{\text {high }}=\left(\frac{f}{\tau_{i}}+\frac{1-f}{\tau_{e}}\right)^{-1} .
$$

The low and high-frequency averages for the relaxation time $\tau$ were observed in numerical experiments that were performed in [4], in which a probabilistic approach is taken to an electromagnetic interrogation problem of a dielectric medium that is a mixture of two Debye media with different relaxation times $\tau$. The low-frequency average corresponds to the average relaxation time that is observed when the composite media is interrogated by electromagnetic pulses at frequencies of $10^{6} \mathrm{~Hz}$, whereas the high-frequency average was observed at higher frequencies of $10^{9}-10^{11} \mathrm{~Hz}$ (frequencies are considered low here if the corresponding angular frequency $\omega<1 / \tau$ ).

In Fig. 7, we also compare the homogenized DRF to the weighted average of the corresponding DRF's, i.e.,

$$
v_{\mathrm{wa}}(t)=f v_{i}+(1-f) v_{e},
$$

where

$$
\begin{aligned}
& \text { (i) } v_{i}(t)=\frac{\epsilon_{0}\left(\epsilon_{s}-\epsilon_{\infty}\right)}{\tau_{i}} \mathrm{e}^{-t / \tau_{i}}, \\
& \text { (ii) } v_{e}(t)=\frac{\epsilon_{0}\left(\epsilon_{s}-\epsilon_{\infty}\right)}{\tau_{e}} \mathrm{e}^{-t / \tau_{e}}
\end{aligned}
$$

as well as to another average that we call the inverse weighted average, which is the inverse of the weighted averages of the inverses of the two DRF's, i.e.,

$$
v_{\text {Iwa }}(t)=\left(\frac{f}{v_{i}}+\frac{(1-f)}{v_{e}}\right)^{-1},
$$



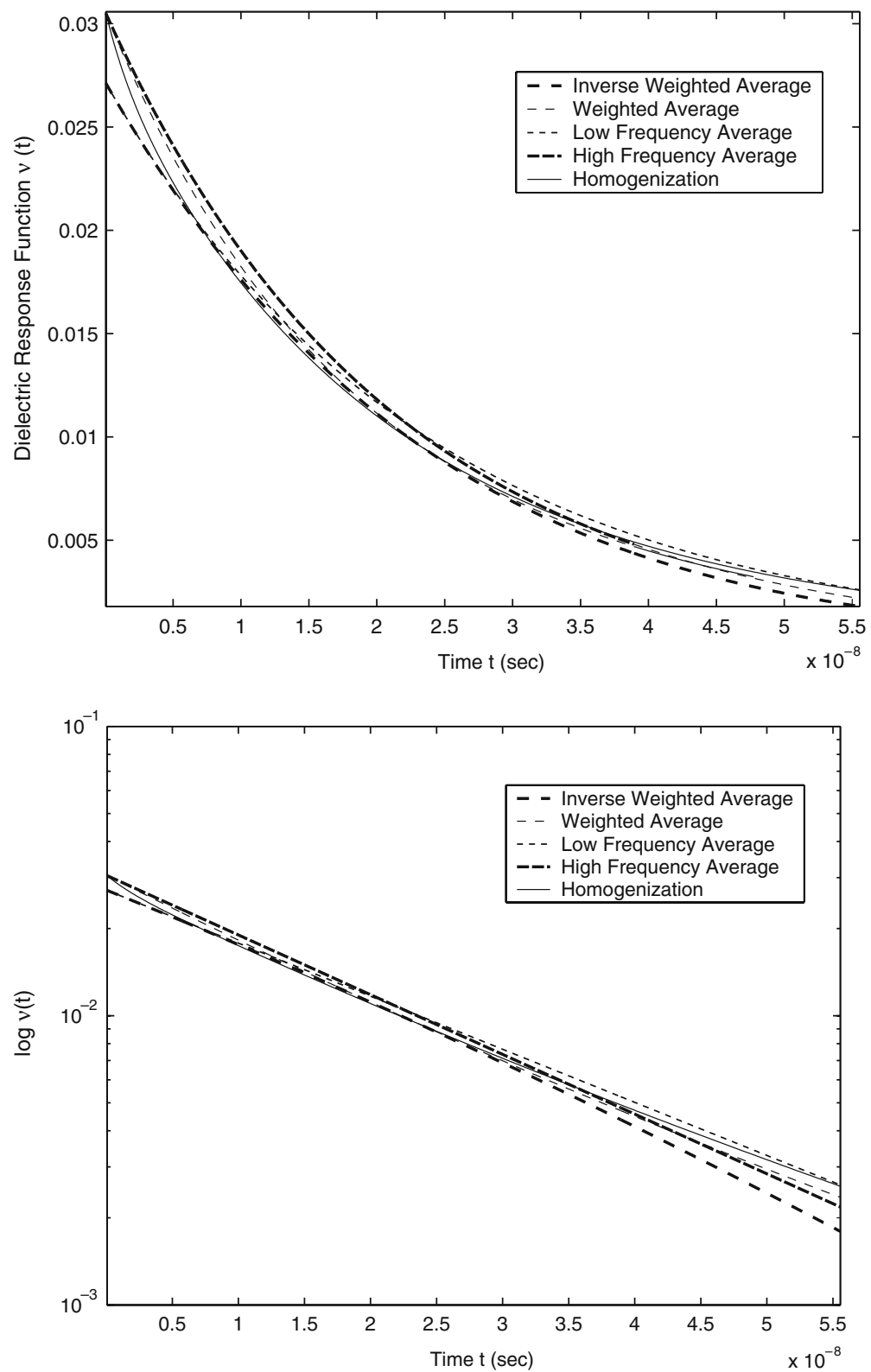

Fig. 7. (top) The effective DRF $v(t)$ as a function of time, for volume fraction $f=0.5$. We compare the homogenized DRF with the DRF that is a weighted average of the corresponding DRF's, as well averages that are observed in other experiments. (bottom) Semilog plot of the DRF's. 
where $v_{i}$ and $v_{e}$ are defined in $(85$, i) and $(85$, ii), respectively. Clearly Fig. 7 demonstrates good agreement between the homogenized DRF and the other approximations. Finally in Fig. 8 we plot the homogenized DRF as a function of the inclusion volume fraction at times $t=0.556$ and $t=27.78 \mathrm{~ns}$. For $t \ll \min \left(\tau_{i}, \tau_{e}\right)$ one can show that $\nu_{\text {Iwa }}(t) \approx v\left(t ; \tau_{\text {low }}\right)$ as is clearly seen in the top plot of this figure. Similarly we have $v_{\mathrm{wa}}(t) \approx$ $v\left(t ; \tau_{\text {high }}\right)$. We observe that for all values of $f$ the plot of the homogenized DRF lies between the other approximations for both small and large times.

\section{CONCLUSIONS AND FUTURE DIRECTIONS}

Taking into account the large deformations of the corrector functions (see Figs. 5 and 6) we can expect that the correction of the electric and magnetic fields as given by (20) will be of crucial interest. The results of the cell problem with circular microstructures for calculating effective relative permittivities agree well with the mixing formulae results that are available in the literature. However, there are several advantages to taking a homogenization approach to obtain effective dielectric parameters. First, mixing formulae are not available for general microstructure geometries. Close examination of the materials of long-term interest to us reveal that the foam consists of hexagonal-like cells in a microstructure configuration that is $95 \%$ gas surrounded by a matrix of polyurethane, which has an estimated relative permittivity of 2.7. A homogenization approach will permit us to ascertain the sensitivity of the effective permittivities with respect to the micro-geometry. While we have used a staircase method to approximate the circular microstructure in the cell problem, the approach also permits better approximations (e.g., a fictitious domain approach) for the circular (and other shape) microstructures in the cell problem.

A second advantage of a homogenization approach is the flexibility it affords in assumptions about material polarization laws. Here in proofof-concept calculations we have used a Debye medium for polarization but could with equal ease investigate the cases of Lorentz media, composite laws and other higher order dispersive media. Moreover, as we investigate other mechanisms, it may be computationally advantageous to treat directly the polarization law as a side constraint. That is, the hysteretic term of the polarization is most generally represented by an integral term, which involves a dielectric response function. Our numerical approximation to this approach involved a recursive convolution method to approximate the integral term. Our current efforts entail an approach in which the homogenized model involves an ordinary differential equation for the hysteretic part of the polarization term. This approach should facilitate 

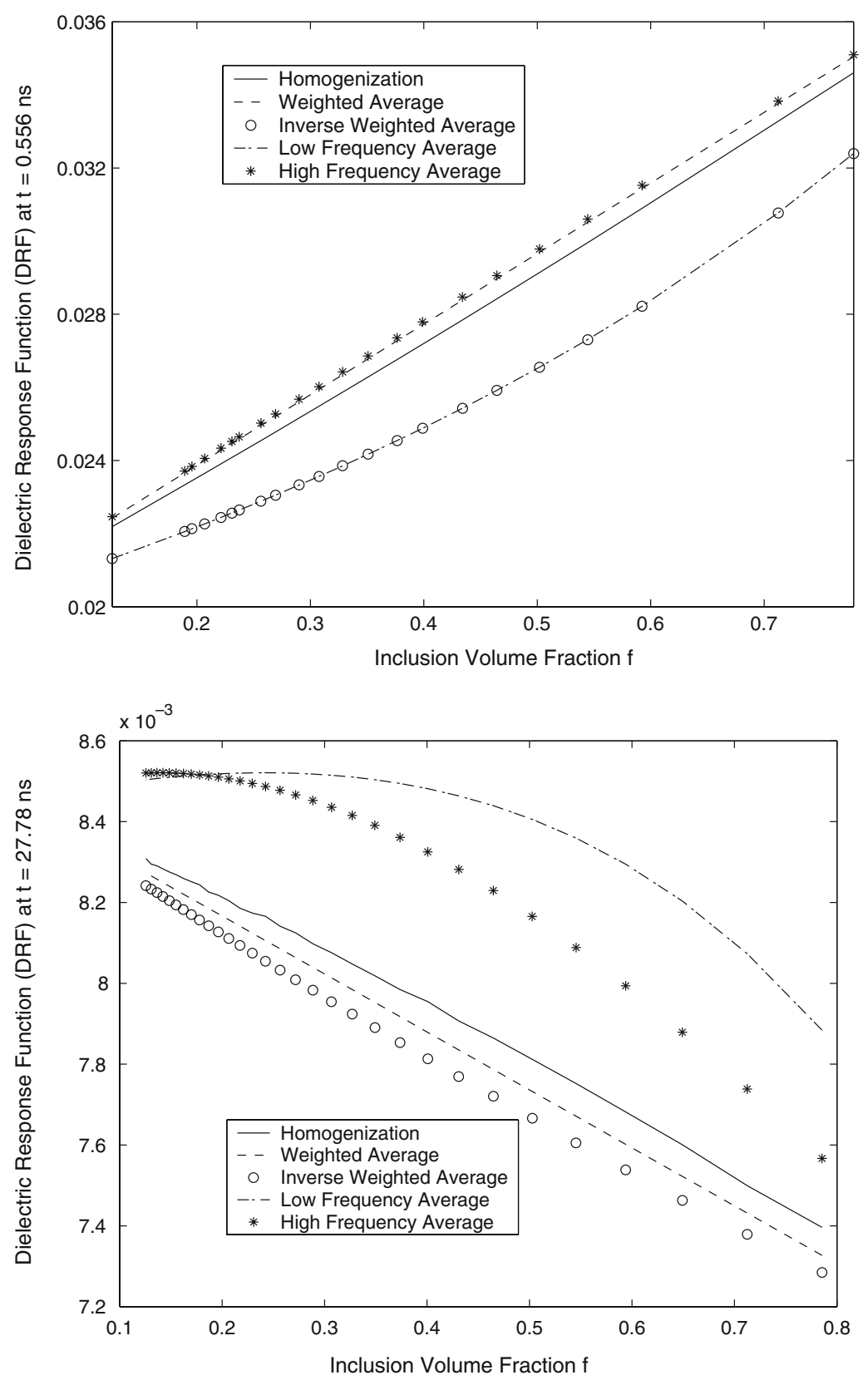

Fig. 8. (top) The effective DRF $v(t)$ as a function of the inclusion volume fraction for $t=0.556 \mathrm{~ns}$ and (bottom) $t=27.78 \mathrm{~ns}$. 
numerical approximation of the solution of the electromagnetic interrogation problem for a wide class of assumed polarization mechanisms.

The initial results of the cell problems for the calculation of the dielectric response function compare well with the results that were obtained in [4], where a probabilistic approach is taken to an electromagnetic interrogation problem in a dielectric medium that is a mixture of two Debye media with different relaxation times. Each approach has its own conceptual, theoretical and computational advantages that merit further comparisons.

\section{ACKNOWLEDGMENTS}

This research was supported in part by the U.S. Air Force Office of Scientific Research under grant AFOSR FA9550-04-1-0220, in part by the National Institute of Aerospace(NIA) and NASA under grant NIA/NCSU-03-01-2536-NC, in part by the European Community's Human Potential Programme under contract HMS 2000 number HPRN-CT-200000109 and contract Smart Systems number HPRN-CT-2002-00284. Collaboration was also facilitated through visits by several of the authors to the Statistical and Applied Mathematical Sciences Institute (SAMSI), Research Triangle Park, NC, which is funded by NSF under grant DMS0112069. The authors would like to thank Dr. William P. Winfree of the NASA Langley Research Center for numerous stimulating and valuable comments and suggestions on this work.

\section{REFERENCES}

1. Allaire, G. (1992). Homogenization and two-scale convergence. SIAM J. Math. Anal. 23(6), 1482-1518.

2. Banks, H. T., Bokil, V. A., Cioranescu, D., Gibson, N. L., Griso, G., and Miara, B. (2005). Homogenization of periodically varying coefficients in electromagnetic materials. CRSC Technical Report, CRSC-TR05-05.

3. Banks, H. T., Buksas, M. W., and Lin, T. (2000). Electromagnetic Material Interrogation Using Conductive Interfaces and Acoustic Wavefronts, Frontiers in Applied Mathematics. v. FR21, SIAM, Philadelphia, PA, 2000.

4. Banks, H. T., and Gibson, N. L. (2005). Inverse problems for Maxwell's equations with distributions of dielectric parameters. CRSC Technical Report CRSC-TR05-29, August 2005, N.C. State University; available at <http://www.ncsu.edu/crsc/>.

5. Banks, H. T., Gibson, N. L., and Winfree, W. P. (2005). Gap detection with electromagnetic Terahertz signals. Nonlin. Anal. Real World Appl. 6, 381-416.

6. Bossavit, A., Griso, G., and Miara, B. (2004). Modélisation de structures électromagnetiques périodiques: matériaux bianisotropiques avec mémoire. C. R. Acad. Sci. Paris, Ser. I. 338, 97-102.

7. Bossavit, A., Griso, G., and Miara, B., Modelling of periodic electromagnetic structures bianisotropic material with memory effects, J. Mathématiques Pures et Appliqu'ees, in press. 
8. Cioranescu, D. (2003). Homogenization and applications to material sciences in Research Directions in Distributed Parameter Systems, Frontiers in Applied Mathematics, 55-94, $v$. FR27, SIAM, Philadelphia, PA.

9. Cioranescu, D., Damlamian, A., and Griso, G. (2002). Periodic unfolding and homogenization. C. R. Acad. Sci. Paris, Ser. I, 335, 99-104.

10. Cioranescu, D., and Donato, P. (1999). An Introduction to Homogenization, Number 17 in Oxford Lecture Series in Mathematics and its Applications. Oxford University Press, Oxford.

11. Cole, K. S., and Cole, R. H. (1941). Dispersion and absorption in dielectrics. J. Chem. Phy. 9, 341-351.

12. Debye, P. (1929). Polar Molecules, Chemical Catalog Co., New York.

13. Engström, C., and Sjöberg, D., (2004). A comparison of two numerical methods for homogenization of Maxwell's equations, Technical Report LUTEDX/(TEAT-7121)/1-10/ (2004), Department of Electroscience, Lund Institute of Technology, Sweden.

14. El Feddi, M., Ren, Z., Razek, A., and Bossavit, A. (1997). Homogenization technique for Maxwell equations in periodic structures. IEEE Trans. Mag. 33(2), 1382-1385.

15. Gibson, N. L. (2004). Terahertz-Based Electromagnetic Interrogation Techniques for Damage Detection, Ph.D. Thesis, N.C. State University, Raleigh, NC.

16. Kelley, D. F., and Luebbers, R. J. (1996). Piecewise linear recursive convolution for dispersive media using FDTD. IEEE Trans. Antennas Propagat. 44(6), 792-797.

17. Kristensson, G. (2001). Homogenization of the Maxwell equations in an anisotropic material. Technical Report LUTEDX/(TEAT-7104)/1-12/(2001), Department of Electroscience, Lund Institute of Technology, Sweden, 2001.

18. Kristensson, G. (2004). Homogenization of corrugated interfaces in electomagnetics. Technical Report LUTEDX/(TEAT-7122)/1-29/(2004), Dept. of Electroscience, Lund Institute of Technology, Sweden.

19. Luebbers, R. J., Hunsberger, F. P., Kunz, K. S., Standler, R. B., and Schneider, M. (1990). A frequency-dependent finite difference time-domain formulation for dispersive materials. IEEE Trans. Elect. Compat. 32(3), 222-227.

20. NASA Facts: Thermal Protection System. NASA Report FS-2004-08-97 MSFC, NASA Marshall Space Flight Center, Huntsville, AL.

21. Nguetseng, G. (1989). A general convergence result for a functional related to the theory of homogenization. SIAM J. Math. Anal. 20(3), 608-623.

22. Ouchetto, O., Zouhdi, S., Bossavit, A., Griso G., and Miara, B. (2005). Homogenization of 3D structured composites of complex shaped inclusions, PIERS 2005. In Progress in Electromagnetics Research Symposium, pp. 22-260. Hangzhou. China.

23. Sihvola, A. (1999). Electromagnetic Mixing Formulas and Applications, IEE Electromagnetic Waves Series, The Institute of Electrical Engineers, London, p. 47.

24. Sihvola, A. (2000). Effective permittivity of mixtures: numerical validation by the FDTD method, IEEE Trans. Geosci. Remote Sensing. 38(3), 1303-1308.

25. Sjöberg, D. (2004). Homogenization of dispersive material parameters for Maxwell's equations using a singular value decomposition. Technical Report LUTEDX/(TEAT-7124)/ 1-24/(2004), Department of Electroscience, Lund Institute of Technology, Sweden.

26. Sjöberg, D., Engström, C., Kristensson, G., Wall, D. J. N., and Wellander, N. (2003). A Floquet-Bloch decomposition of Maxwell's equations, applied to homogenization. Technical Report LUTEDX/(TEAT-7119)/1-27/(2003), Department of Electroscience, Lund Institute of Technology, Sweden.

27. Wellander, N., and Kristensson, G. (2002). Homogenization of the Maxwell equations at fixed frequency. Technical Report LUTEDX/(TEAT-7103)/1-38/(2001), Department of Electroscience, Lund Institute of Technology, Sweden. 